\title{
EQUIVALENCE OF LOW FREQUENCY STABILITY CONDITIONS FOR MULTIDIMENSIONAL DETONATIONS IN THREE MODELS OF COMBUSTION
}

\author{
HELGE KRISTIAN JENSSEN, GREGORY LYNG, MARK WILLIAMS
}

\begin{abstract}
We use the classical normal mode approach of hydrodynamic stability theory to define stability determinants (Evans functions) for multidimensional strong detonations in three commonly studied models of combustion: the full reactive Navier-Stokes (RNS) model, and the simpler Zeldovich-von Neumann-Döring (ZND) and Chapman-Jouguet (CJ) models. The determinants are functions of frequencies $(\lambda, \eta)$, where $\lambda$ is a complex variable dual to the time variable, and $\eta \in \mathbb{R}^{d-1}$ is dual to the transverse spatial variables. The zeros of these determinants in $\Re \lambda>0$ correspond to perturbations that grow exponentially with time.

The CJ determinant, $\Delta_{C J}(\lambda, \eta)$, turns out to be explicitly computable. The RNS and ZND determinants are impossible to compute explicitly, but we are able to compute their first-order low frequency expansions with an error term that is uniformly small with respect to all possible $(\lambda, \eta)$ directions. Somewhat surprisingly, this computation yields an Equivalence Theorem: the leading coefficient in the expansions of both the RNS and ZND determinants is a constant multiple of $\Delta_{C J}$ ! In this sense the low frequency stability conditions for strong detonations in all three models are equivalent. By computing $\Delta_{C J}$ we are able to give low frequency stability criteria valid for all three models in terms of the physical quantities: Mach number, Gruneisen coefficient, compression ratio, and heat release. The Equivalence Theorem and its surrounding analysis is a step toward the rigorous theoretical justification of the CJ and ZND models as approximations to the full RNS model.
\end{abstract}

\section{Contents}

Part 1. Introduction 1

$\begin{array}{llr}\text { Part 2. } & \text { Three models of combustion } & 7\end{array}$

Part 3. Profiles and Evans functions 10

1. Steady detonation profiles 10

2. Assumptions 12

3. Linearization $\quad 15$

4. Stability determinants 19

Part 4. The Equivalence Theorem 30

5. Low frequency expansion for ZND 31

6. Low frequency expansion for RNS 33

$\begin{array}{lll}\text { Part 5. The physical equations } & 38\end{array}$

Date: July 13, 2004: revised: August 24, 2004. 
7. Evaluation of $\Delta_{C J} \quad 38$

8. The transversality coefficients $\beta_{1}$ and $\beta_{2} \quad 44$

9. The case of an ideal polytropic gas 46

Part 6. Appendix: Well-posedness of the combustion equations 46

$\begin{array}{ll}\text { References } & 48\end{array}$

\section{Part 1. Introduction}

It is well-known that detonation waves, in spite of their similarities to nonreactive shocks, exhibit a wider variety of instabilities than shocks. For example, detonation waves propagating in gas-filled tubes can display "galloping" and "spinning" structures. The surface of a planar detonation front often has a cellular structure [FD] consisting of transverse waves which travel across the front. Observations show that these are unsteady structures which fluctuate, decaying until they are reinvigorated by collisions with other such waves. This rich variety of behaviors indicates the multidimensional nature of detonation fronts and the complexity of the stability problem.

In this paper we study the spectral stability of strong detonation fronts in the model of reactive flow given by the full reactive Navier-Stokes equations (RNS), as well as in two of the most thoroughly studied simplified models, the Zeldovich-von Neumann-Döring (ZND), and Chapman-Jouguet (CJ) models. The RNS model consists of the compressible Navier-Stokes equations coupled to a reaction equation; the model takes account of effects due to viscosity and heat conductivity in addition to species diffusion, reaction rate, and heat release. In the ZND model the dissipative effects of viscosity, heat conductivity, and species diffusion are neglected (at times we'll refer to the combination of these dissipative effects as "viscosity"), but the reaction is still assumed to proceed at a finite rate. Thus, the system looks like the Euler equations coupled to a reaction equation. Finally, in the CJ model the reaction is assumed to proceed instantaneously, so the reaction equation is eliminated entirely and we are left with Euler equations in which the internal energy terms change discontinuously across the front that is the boundary between completely burnt and unburnt gas.

The three models are described precisely in Part 2. We first describe the actual physical equations and then generalize to abstract models that contain the physical equations as special cases. We formulate structural hypotheses for the abstract models in Part 3, and then prove our main Equivalence Theorem (Theorem 0.1 below) in the abstract setting. Every abstract structural assumption is satisfied by the corresponding physical equation. We work with the abstract models not just in order to generalize; in fact, they make it easier to see what structural features of the physical equations are really important for the purposes at hand.

For each of the three models we apply the classical approach of hydrodynamic stability theory (e.g., [Ch, DR, FD]) to study the stability of steady planar detonation fronts. That is, in each case we linearize the system about a steady solution and look for oscillatory "normal mode" solutions to the linearized problem of the form

$$
N\left(t, y^{\prime}, x\right)=e^{\lambda t+i y^{\prime} \eta} n(x, \lambda, \eta)
$$


where the surfaces $x=$ constant are parallel to the front, $y^{\prime}=\left(y_{1}, \ldots, y_{d-1}\right)$ are the transverse variables, $t$ is time, and $\lambda=i \tau+\gamma, \eta$ are dual variables with $(\tau, \eta) \in \mathbb{R}^{d}$ and $\gamma \geq 0$. Solutions (0.1) with $\gamma>0$ and $n$ decaying as $x \rightarrow \pm \infty$ correspond to multidimensional perturbations that grow exponentially with time. The spectral stability problem is to identify the locations in frequency space (i.e., $(\lambda, \eta)$-space) where exponentially growing perturbations do or do not exist. For each model we define an associated determinant, denoted $D_{R N S}(\lambda, \eta)$, $D_{Z N D}(\lambda, \eta)$, or $\Delta_{C J}(\lambda, \eta)$, which vanishes precisely at those frequencies $(\lambda, \eta)$ where exponentially growing perturbations exist. Thus, solving the spectral stability problem for a given model is equivalent to locating the zeros of the corresponding determinant.

The function $D_{R N S}(\lambda, \eta)$ is an Evans function, a Wronskian of decaying solutions to the linearized equations, while $\Delta_{C J}$ is a Lopatinski determinant, much like the stability determinants defined by Kreiss $[\mathrm{K}]$ for hyperbolic boundary problems or Majda [Ma1] for nonreactive shocks. The function $D_{Z N D}$, which we'll sometimes refer to as the ZND Evans function, is really a combination of the two kinds of determinants.

The spectral approach to stability questions in combustion was initiated by Erpenbeck [E1, E2, E3, E4, E5, E6] for the ZND model in the 1960s (a summary of this work is given in $[\mathrm{FD}]$ ). He defined a "stability function" (called $V(\lambda, \eta)$ in [E1]) in the frequency domain $\{(\lambda, \eta): \Re \lambda>0,|\eta|>0\}$, whose zeros coincide with those of our $D_{Z N D}$ in that domain.

A major difficulty with the spectral stability problem for the RNS and ZND models is that the steady solutions in those cases are travelling-wave profiles

$$
\begin{aligned}
& V_{R N S}(x) \rightarrow V_{ \pm} \text {as } x \rightarrow \pm \infty, \text { or } \\
& V_{Z N D}(x) \rightarrow V_{ \pm} \text {as } x \rightarrow \pm \infty
\end{aligned}
$$

so the corresponding linearized problem for $n(x, \lambda, \eta)$ is in each case a complicated system of nonautonomous ODEs in $x$ depending on frequencies as parameters. It is precisely because of this difficulty that much of the research on the detonation stability problem relies on numerical computations. Following Erpenbeck, Fickett \& Wood [FW] initiated a series of numerical investigations that continues up to the present day (e.g., [AT, LS]).

Remark 0.1. The profile $V_{R N S}(x)$ is smooth on $(-\infty,+\infty)$, while $V_{Z N D}(x)$ has a discontinuity at $x=0$, often called the von Neumann jump, which reflects the neglect of dissipative effects in the ZND model.

In addition to the numerical investigations, various asymptotic regimes have been considered. Erpenbeck [E5] treated the case of high frequency perturbations, while weak heat release and high activation energy have been considered by [SS, Sh, AT, BN] among others. Majda, Bourlioux, Colella, and Roytburd [BM1, BM2, CMR] use a combination of theoretical, asymptotic, and numerical ideas to study the structure of detonations. In this paper we give the first theoretical treatment of the low frequency regime $|\lambda, \eta|<\delta$ that is uniformly valid as $\Re \lambda \rightarrow 0$.

In the CJ model we linearize about the steady solution given by the constant endstates $V_{ \pm}$in (0.2). Thus, we obtain a much simpler system of ODEs for $n(x, \lambda, \eta)$ in this case, and it turns out that the determinant $\Delta_{C J}(\lambda, \eta)$ is explicitly computable in terms of measurable physical quantities. One of the main results of this paper, the Equivalence Theorem, implies that $\Delta_{C J}$ can in fact be used to deduce information about $D_{R N S}$ and $D_{Z N D}$ in the low frequency regime $|\lambda, \eta|<\delta$ corresponding to long-wavelength perturbations. Here, of course, 
we assume that the constant states defining the steady CJ solution are the endstates as in (0.2) for both the RNS and ZND profiles. The low frequency regime is the only regime where we can reasonably expect agreement of spectral stability conditions for the three models (see part 3 of Remark 0.3).

In order to describe this theorem we first introduce polar coordinates

$$
\zeta=\rho \hat{\zeta}=\rho(\hat{\tau}, \hat{\gamma}, \hat{\eta}), \rho=|\zeta|
$$

(sometimes we also write $\zeta=\rho(\hat{\lambda}, \hat{\eta}))$, where

$$
\hat{\zeta} \in S_{+}^{d}=\left\{\hat{\zeta}=(\hat{\tau}, \hat{\gamma}, \hat{\eta}):(\hat{\tau}, \hat{\eta}) \in \mathbb{R}^{d},|\hat{\zeta}|=1, \hat{\gamma} \geq 0\right\}
$$

Thus, when $|\lambda, \eta|>0$ we may unambiguously write

$$
D_{R N S}(\lambda, \eta)=D_{R N S}(\hat{\zeta}, \rho),
$$

and do similarly for the other two determinants. In fact, $\Delta_{C J}(\lambda, \eta)$ is homogeneous of degree one in $|\lambda, \eta|>0$, so we have

$$
\Delta_{C J}(\hat{\zeta}, \rho)=\rho \Delta_{C J}(\hat{\zeta}, 1)
$$

The determinants $D_{R N S}(\hat{\zeta}, \rho)$ and $D_{Z N D}(\hat{\zeta}, \rho)$ are not homogeneous in $\rho>0$, but both are readily shown to vanish to at least first order in $\rho$ as $\rho \rightarrow 0$.

The three determinants are first defined in the frequency domain $\{(\hat{\zeta}, \rho): \hat{\gamma}>0, \rho>0\}$, where they are $C^{\infty}$ (in fact, analytic) functions. An essential prerequisite for a uniform stability analysis in the low frequency regime is to construct continuous extensions of

$$
\frac{D_{R N S}(\hat{\zeta}, \rho)}{\rho}, \frac{D_{Z N D}(\hat{\zeta}, \rho)}{\rho}, \text { and } \Delta_{C J}(\hat{\zeta}, 1)
$$

to the domain

$$
\{(\hat{\zeta}, \rho): \hat{\gamma} \geq 0, \rho \geq 0\}
$$

Let us briefly indicate how this is done in the case of the RNS system. Imagine that the second order linearized problem is rewritten as a first-order system:

$$
U_{x}-G(x, \hat{\zeta}, \rho) U=0,
$$

and consider the associated constant-coefficient limiting problems

$$
U_{x}-G_{ \pm}(\hat{\zeta}, \rho) U=0
$$

obtained by letting $x \rightarrow \pm \infty$ in $G(x, \hat{\zeta}, \rho)$ (recall $(0.2)$ ). Let $E_{ \pm}(\hat{\zeta}, \rho)$ denote the vector spaces consisting of initial data at $x=0$ of solutions of $(0.9)$ that decay as $x \rightarrow \pm \infty$. We note that $D_{R N S}(\hat{\zeta}, \rho)$ may be defined for $\rho>0$, up to nonvanishing factors, simply as

$$
\operatorname{det}\left(E_{+}(\hat{\zeta}, \rho), E_{-}(\hat{\zeta}, \rho)\right)
$$

(the dimensions of $E_{ \pm}$work out so that the determinant is well-defined). Constructing the desired continuous extension of the first function in (0.7) now reduces to continuously extending the spaces $E_{ \pm}(\hat{\zeta}, \rho)$. Note that we can define analogous spaces $F_{ \pm}(\hat{\zeta}, \rho)$ for the limiting problems $(0.10)$.

A conjugation argument first given in [MZ1] and used repeatedly in [GMWZ1, GMWZ2, GMWZ3] allows us to reduce the study of (0.9) to the much simpler problem (0.10). Thus, 
continuous extension of the spaces $E_{ \pm}$reduces to continuous extension of $F_{ \pm}$. For $\rho>0$ $F_{ \pm}(\hat{\zeta}, \rho)$ is just the sum of the generalized eigenspaces of $G_{ \pm}(\hat{\zeta}, \rho)$ corresponding to eigenvalues $\mu(\hat{\zeta}, \rho)$ with $\mp \Re \mu>0$. Thus, it is easy to obtain continuous extensions near points $(\hat{\zeta}, \rho)$ with $\hat{\gamma}=0$ and $\rho=0$ in the cases when $G_{ \pm}(\hat{\zeta}, \rho)$ has no pure imaginary eigenvalues, or when its only pure imaginary eigenvalues are simple. The main difficulties occur near glancing points, which are by definition points $(\hat{\zeta}, \rho)$ where $G_{ \pm}(\hat{\zeta}, \rho)$ has at least one pure imaginary eigenvalue of multiplicity $\geq 2$. They constitute a proper subset of $\{\rho=0, \hat{\gamma}=0\}$. The problem is to understand how eigenvalues and eigenspaces vary as the two parameters $\hat{\gamma}$ and $\rho$ are perturbed to positive values near these points. Observe that glancing points are a special feature of multidimensional problems; they do not occur in 1D.

The extension in the CJ case, where the parameter $\rho$ plays no role, easily follows from the work of Kreiss [K] and Majda [Ma1]. The extensions in the (two-parameter) ZND and RNS cases present new difficulties, many of which are identical to those encountered in [MZ1, MZ2, GMWZ3], where it is shown how to construct continuous extensions of the decaying eigenspaces that arise in the study of viscous boundary layers and nonreactive viscous shocks. In Part 3 we define the three combustion determinants and give the conjugation argument (specifically, conjugation of the limiting system (0.10) to a block diagonal form $G_{B \pm}$; see Proposition 4.4) needed to place the extension problem for the RNS and ZND determinants into the framework of [MZ1, MZ2, GMWZ3]. The references [MZ1, GMWZ3] show how to construct Kreiss-type symmetrizers for the limiting system in $G_{B \pm}$ form. Those symmetrizers (which have in the past just been used to obtain linearized stability estimates) can then be used as in [MZ2] to obtain continuous extensions of decaying eigenspaces.

Remark 0.2. The analysis of this paper makes no explicit use of Kreiss symmetrizers. Once we show that the limiting RNS and ZND systems can be put in $G_{B \pm}$ form, we just cite the above references to obtain continuous extensions of decaying eigenspaces.

With the continuous extensions in hand, we are now in a position to derive uniform low frequency expansions for $D_{R N S}$ and $D_{Z N D}$. This is done in part 4 , where we prove the Equivalence Theorem (see Theorem 6.2 for a precise statement):

Theorem 0.1. Assume that the states $V_{ \pm}$define a strong detonation solution to the ChapmanJouguet system with Lax n-shock structure (See Definition 1.1 below). Then

$$
\begin{aligned}
& \text { (a) } D_{Z N D}(\hat{\zeta}, \rho)=\rho \beta_{1} \Delta_{C J}(\hat{\zeta}, 1)+O\left(\rho^{2}\right) \\
& \text { (b) } D_{R N S}(\hat{\zeta}, \rho)=\rho \beta_{2} \Delta_{C J}(\hat{\zeta}, 1)+o(\rho)
\end{aligned}
$$

for constants $\beta_{1}$ and $\beta_{2}, \hat{\zeta} \in S_{+}^{d}=\{(\lambda, \eta):|\lambda, \eta|=1, \Re \lambda \geq 0\}$, and $\rho=|\lambda, \eta| \geq 0$. The errors are $O\left(\rho^{2}\right)$ (respectively, o( $\left.\rho\right)$ ) uniformly for $\hat{\zeta} \in S_{+}^{d}$. The constants $\beta_{1}, \beta_{2}$ are nonvanishing precisely when the corresponding profiles define transverse connections.

A key point is that the same factor $\Delta_{C J}(\hat{\zeta}, 1)$ appears in both (a) and (b) of $(0.12)$. Thus, $\Delta_{C J}$ contains the essential information about low frequency stability for all three models, and so in this sense the low frequency spectral stability conditions in all three models are equivalent. The theorem can be viewed as a step toward the rigorous theoretical justification of the CJ and ZND models as approximations to the more complete RNS model. 
An important consequence of the Equivalence Theorem is that transversality and nonvanishing of $\Delta_{C J}(\hat{\zeta}, 1)$ (immediately) imply precisely first order vanishing of $D_{R N S}$ and $D_{Z N D}$ at $\rho=0$, uniformly with respect to $\hat{\zeta} \in S_{+}^{d}$. In turn this yields

$$
\begin{aligned}
& D_{R N S}(\hat{\zeta}, \rho) \neq 0 \text { for } 0<\rho<\delta \\
& D_{Z N D}(\hat{\zeta}, \rho) \neq 0 \text { for } 0<\rho<\delta
\end{aligned}
$$

for $\delta$ small enough and independent of $(\hat{\zeta}, \rho)$. This result can be used to rule out violent low frequency instabilities for $0<\rho<\delta$ and also weaker instabilities where $\hat{\gamma}$ might be 0 .

Another consequence of Theorem 0.1 is that, under certain circumstances (e.g., assuming "one-dimensional" stability: $\beta_{i} \Delta_{C J} \neq 0$ when $\left.\hat{\eta}=0\right)$ nonvanishing of $\Delta_{C J}(\hat{\zeta}, 1)$ in $\hat{\gamma}>0$ is a necessary condition for nonvanishing of $D_{R N S}(\hat{\zeta}, \rho)$ or $D_{Z N D}(\hat{\zeta}, \rho)$ in $\gamma=\rho \hat{\gamma}>0$ (see [Z], Lemma 3.2 for a nearby argument). In other words absence of violent CJ instabilities is a necessary condition for absence of violent RNS or ZND instabilities.

In part 5 we compute $\Delta_{C J}$ and the transversality coefficients $\beta_{1}, \beta_{2}$ for the physical equations. We characterize precisely when $\Delta_{C J}(\hat{\zeta}, 1)$ has:

(a) a zero with $\hat{\gamma}>0$

(b) a zero with $\hat{\gamma}=0$ but none with $\hat{\gamma}>0$,

(c) no zeros with $\hat{\gamma} \geq 0$,

in terms of the physical quantities: Mach number, Gruneisen coefficient, compression ratio, and heat release. Using this result, we show that strong detonations occurring in an ideal polytropic gas are always uniformly low frequency stable; that is, $\Delta_{C J}$ is nonvanishing on $S_{+}^{d}$.

The local well-posedness in time of the RNS and ZND models is far from immediately obvious; especially in the case of the RNS model with its degenerate (or "real") viscosity. Kawashima $[\mathrm{Ka}]$ constructed symmetrizers for the nonreactive Navier-Stokes equations that yield local well-posedness in time by a simple integration by parts argument. In the Appendix, we write down a Kawashima-type symmetrizer that works in the same way for the RNS and ZND models. It turns out one can do this by starting with a Kawashima symmetrizer and adding an appropriate row and column corresponding to the reaction equation.

Remark 0.3. 1. As far as we know the first low frequency expansion of an Evans function of the type that appears in (0.12)(a), for example, was given for 1D viscous shocks in GardnerZumbrun [GZ]. The first such expansion for multidimensional (nonreactive) viscous shocks appears in the pioneering paper Zumbrun-Serre [ZS]. There, one has an Evans function $D(\hat{\zeta}, \rho)$ for a planar viscous shock, and in place of $\Delta_{C J}$ one has the Majda [Ma1] uniform stability determinant $\Delta(\hat{\zeta}, 1)$ for the corresponding inviscid shock. The analogue of equation $(0.12)(\mathrm{a})$ in that setting,

$$
D(\hat{\zeta}, \rho)=\beta \rho \Delta(\hat{\zeta}, 1)+o(\rho),
$$

can be viewed as asserting the commutativity of low frequency and vanishing viscosity limits (the dependence of the functions $D$ and $D_{R N S}$ on viscosity has been suppressed in the notation). However, the analysis of [ZS] assumes $\hat{\gamma}>0$, and is not valid uniformly for $\hat{\gamma} \rightarrow 0$. Without such a uniform analysis one cannot deduce statements like (0.13), assuming transversality and nonvanishing $\Delta$. Instead, one can only make a weaker statement where $\delta$ depends on $(\hat{\zeta}, \rho)$ and $\delta(\hat{\zeta}, \rho)$ may vanish as $\hat{\gamma} \rightarrow 0$ for some $(\hat{\tau}, \hat{\eta})$. 
A similar problem is present in the discussion of the stability function $V(\lambda, \eta)$ defined for the ZND model by Erpenbeck in [E1]. $V(\lambda, \eta)$ is defined just for $\Re \lambda>0$, and there is no uniform analysis of its behavior as $\hat{\gamma} \rightarrow 0$. As far as we know, Erpenbeck made no attempt to compute a low frequency expansion of $V$; but in a later paper, he studied the high frequency limit [E5].

2. A partial proof of the low frequency expansion (0.12)(a) for the RNS model with "artificial" (positive) viscosity was sketched in Appendix A3 of Zumbrun [Z]. In the RNS case, our main contributions are to extend the argument to the case of "real" viscosity (only partially positive, because viscosity does not appear in the conservation of mass equation), and to provide an analysis that is uniformly valid as $\hat{\gamma} \rightarrow 0$ (not discussed in [Z]).

There is also an attempt in Appendix A3 of $[\mathrm{Z}]$ to derive a low frequency expansion for $D_{Z N D}$. An equation of the form $(0.12)(\mathrm{b})$ is derived there, but where $\Delta_{C J}$ is replaced by a "nonequivalent" Lopatinski determinant $\tilde{\Delta}$ (nonequivalent in the sense that there is no reason to expect the two determinants to have the same zero sets). This discrepancy between the RNS and ZND low frequency expansions was interpreted there to reflect the noncommutativity of low frequency and small viscosity limits for the RNS model. The reason for the discrepancy turns out to be that [Z] worked with an incorrect expression for $D_{Z N D}$. Theorem 0.1 shows that if one starts with the correct ZND Evans function, there is no discrepancy.

3. The equalities in Theorem 0.1 are at first sight rather remarkable, since RNS profiles are close to ZND profiles only when viscosity is small compared to width of the reaction zone (see [GS]). Yet the determinant $\Delta_{C J}$ is independent of viscosity and size of the reaction zone. On the other hand, one might expect such a result since very low frequency (i.e., very long wavelength) perturbations should, roughly speaking, not even "see" the von Neumann jump in the ZND profile or the region of finite extent where the ZND and RNS profiles differ appreciably. The proof of Theorem 0.1 confirms this vague expectation.

\section{Part 2. Three models of combustion}

We set $y_{d}=x$ and denote spatial directions by $\left(y_{1}, \ldots, y_{d}\right)$. The reactive Navier-Stokes (RNS) equations for a $d$-dimensional reacting fluid with a one-step exothermic reaction are given in Eulerian coordinates as (see, e.g., [Wi])

$$
\begin{gathered}
\rho_{t}+\operatorname{div}(\rho \mathbf{u})=0, \\
\left(\rho u_{j}\right)_{t}+\operatorname{div}\left(\rho u_{j} \mathbf{u}\right)+p_{y_{j}}=\epsilon \Delta u_{j}+\nu \operatorname{div}\left(\mathbf{u}_{y_{j}}\right), \quad j=1, \ldots d, \\
(\rho \tilde{E})_{t}+\operatorname{div}[(\rho \tilde{E}+p) \mathbf{u}]=\Delta\left(\kappa T+\epsilon \frac{|\mathbf{u}|^{2}}{2}\right)+\epsilon \operatorname{div}((\nabla \mathbf{u}) \mathbf{u})+ \\
(\nu-\epsilon) \operatorname{div}((\operatorname{div} \mathbf{u}) \mathbf{u})+\operatorname{div}(q \rho \beta \nabla Y), \\
(\rho Y)_{t}+\operatorname{div}(\rho Y \mathbf{u})=\operatorname{div}(\rho \beta \nabla Y)-k \rho Y \phi(T) .
\end{gathered}
$$

Here the unknowns are $(\rho, \mathbf{u}, T, Y)$ and the system has dimension $(n+s) \times(n+s)$, where

$$
n=d+2, s=1 .
$$

(Later we'll consider a more complicated multi-step model in which several species of gas are involved, so in that case $s>1$.) 
The divergence is taken with respect to the spatial directions and the matrix $\nabla \mathbf{u}$ in $(0.15 \mathrm{c})$ denotes the Jacobian matrix of the velocity vector with respect to the spatial variables. We write

$$
\tilde{E}=\tilde{e}+\frac{|\mathbf{u}|^{2}}{2}
$$

and our labels are given in the table below.

\begin{tabular}{|ll|}
\hline$\rho$ & density \\
$p$ & pressure \\
$\mathbf{u}=\left(u_{1}, \ldots, u_{d}\right)^{\text {tr }}$ & fluid velocity \\
$T$ & temperature \\
$\tilde{e}$ & specific internal energy \\
$Y$ & mass fraction of reactant \\
\hline$\nu, \epsilon$ & viscosity coefficients \\
$\kappa$ & heat conductivity \\
$\beta$ & species diffusion \\
\hline$k$ & reaction rate \\
$q$ & heat release \\
\hline
\end{tabular}

The quantities $\epsilon, \nu, \kappa, \beta, k$, and $q$ are assumed to be positive constants. We note that the assumption $q>0$ corresponds to an exothermic reaction. In this case we also write

$$
\tilde{e}=e+q Y, \quad \text { where } e=c_{v} T,
$$

and we write $E:=e+|\mathbf{u}|^{2} / 2$. We further assume that the pressure is a given function of the density and the gas-dynamic specific internal energy, so

$$
p=p(\rho, e) \text {. }
$$

Finally, the smooth, increasing function function $\phi(T)$ is the ignition function. We make the standard assumption that $\phi$ satisfies ignition temperature kinetics, that is,

$$
\phi(T)=\left\{\begin{array}{l}
0, \text { for } T<T_{i} \\
1, \text { for } T>T_{0}>T_{i}
\end{array} .\right.
$$

Thus, $\phi$ serves to turn on the reaction in equation $(0.15 \mathrm{~d})$.

Definition 0.1. To obtain the ZND equations from the RNS equations, simply set the constants $\epsilon, \nu, \kappa$ and $\beta$ equal to zero in (0.15a)-(0.15d). To obtain the CJ equations from the ZND equations, we eliminate the reaction equation $(0.15 \mathrm{~d})$, and in the energy equation (0.15c) define $\tilde{e}=e+q$ in the unburned gas (where $Y=1$ ) and $\tilde{e}=e$ in the burnt gas $(Y=0)$.

Subtracting $q \cdot(0.15 \mathrm{~d})$ from equation $(0.15 \mathrm{c})$ we obtain,

$$
\begin{aligned}
(\rho E)_{t}+\operatorname{div}[(\rho E+p) \mathbf{u}]=\Delta\left(\kappa T+\epsilon \frac{|\mathbf{u}|^{2}}{2}\right)+ & \\
& \epsilon \operatorname{div}((\nabla \mathbf{u}) \mathbf{u})+(\nu-\epsilon) \operatorname{div}((\operatorname{div} \mathbf{u}) \mathbf{u})+q k \rho Y \phi(T)
\end{aligned}
$$

If we denote the gas-dynamical variables by $V=(\rho, \mathbf{u}, T)$, and set

$$
w=(V, Y), V \in \mathbb{R}^{n}, Y \in \mathbb{R}^{s}
$$


and $y_{0}=t$, we see that the system $(0.15 \mathrm{a}),(0.15 \mathrm{~b}),(0.20),(0.15 \mathrm{~d})$ is a special case of the following abstract model corresponding to an $s$-step exothermic reaction:

$$
\sum_{j=0}^{d} F^{j}(w)_{y_{j}}=\sum_{j, k=1}^{d}\left(B^{j k}(w) w_{y_{k}}\right)_{y_{j}}+R(w) .
$$

Here, corresponding to the decomposition $w=(V, Y)$, we have

$$
F^{j}(w)=\left(\begin{array}{c}
f^{j}(V) \\
g^{j}(V) Y
\end{array}\right), j=0, \ldots, d,
$$

where $f^{j} \in \mathbb{R}^{n}, g^{j} \in \mathbb{R}^{1}$. We split the variable $V$

$$
V=\left(V_{1}, V_{2}\right), V_{1} \in \mathbb{R}^{n-r}, V_{2} \in \mathbb{R}^{r}
$$

(for the physical equations, $V_{1}=\rho, V_{2}=(\mathbf{u}, T)$ ), and the matrices $B^{j k} \in \mathbb{R}^{(n+s) \times(n+s)}$ have the form

$$
B^{j k}(w)=\left(\begin{array}{cc}
\mathcal{B}^{j k}(V) & 0 \\
0 & D^{j k}(V)
\end{array}\right)=\left(\begin{array}{ccc}
0 & 0 & 0 \\
0 & b^{j k}(V) & 0 \\
0 & 0 & D^{j k}(V)
\end{array}\right)
$$

where $\tilde{B}^{j k} \in \mathbb{R}^{n \times n}, b^{j k} \in \mathbb{R}^{r \times r}, D^{j k} \in \mathbb{R}^{s \times s}$. The forcing term is

$$
R(w)=\left(\begin{array}{c}
Q K \psi(V) Y \\
-K \psi(V) Y
\end{array}\right)
$$

where $\psi \in \mathbb{R}^{1}, Q \in \mathbb{R}^{n \times s}$ and $K \in \mathbb{R}^{s \times s}$ are constant matrices, and

(a) $K$ is positive definite,

(b) the first $n-r$ rows of $Q$ are 0 .

For the one-step physical equations we have, for example,

$$
\begin{aligned}
& g^{0}(V)=\rho, g^{j}(V)=\rho u_{j}, j=1, \ldots, d \\
& D^{j k}(V)=\beta \rho \delta_{j k}(\text { Kronecker delta }) \\
& \psi(V)=\rho \phi(T) \\
& f^{0}(V)=\left(\begin{array}{lll}
\rho & \rho \mathbf{u} & \left.\rho\left(c_{v} T+\frac{|\mathbf{u}|^{2}}{2}\right)\right)^{\operatorname{tr}}, \\
Q=(0, \ldots, 0, q)^{\operatorname{tr}}
\end{array}\right.
\end{aligned}
$$

so the first $n-1$ rows of $Q$ are 0 (not just the first $n-r$ ).

Remark 0.4. In an $s$-step reaction, the $\mathrm{y}_{j}$ component of $Y \in \mathbb{R}^{s}$ represents the mass fraction of the $j$-th reactant, with $\mathrm{y}_{j} \in[0,1]$ and $\mathrm{y}_{j}=1$ (resp. 0) corresponding to the completely unburnt (resp., burnt) state. The completely burnt and unburnt states are represented by

$$
\mathbf{0}=(0, \ldots, 0) \in \mathbb{R}^{s}, \mathbf{1}=(1, \ldots, 1) \in \mathbb{R}^{s},
$$


respectively. The $n \times s$ matrix $Q$ in this case has the form

$$
Q=\left(\begin{array}{ccc}
0 & \ldots & 0 \\
\vdots & \ldots & \vdots \\
0 & \ldots & 0 \\
q_{1} & \ldots & q_{s}
\end{array}\right)
$$

where $q_{j}$ denotes the heat released in the $j$ th reaction, $q_{j}>0$ in the case of an exothermic reaction.

Definition 0.2. The abstract model for the ZND equations has the same form as $(0.22)$ with all $B^{j k}$ set equal to 0 . $\tilde{f}^{j}:$

The abstract CJ model has all $B^{j k}=0, R(w)=0$, and all $g^{j}=0$, but $f^{j}$ is replaced by

$$
\sum_{j=0}^{d} \tilde{f}^{j}(V)_{y_{j}}=0
$$

where (recall (0.15a)-(0.15d))

$$
\tilde{f}^{j}(V)=\left\{\begin{array}{l}
f^{j}(V), \text { in the burnt gas } \\
f^{j}(V)+g^{j}(V) Q \mathbf{1}, \text { in the unburnt gas }
\end{array} .\right.
$$

\section{Part 3. Profiles and Evans functions}

\section{Steady Detonation PROFiles}

1.1. The CJ solution. The steady solution for the CJ system (0.30) is given by a pair of constant states

$$
w_{+}=\left(V_{+}, \mathbf{1}\right) \text { in } x>0, w_{-}=\left(V_{-}, \mathbf{0}\right) \text { in } x<0,
$$

satisfying the Rankine-Hugoniot condition at $x=0$ (which expresses conservation of mass, momentum, and energy for the physical equations):

$$
f^{d}\left(V_{+}\right)+g^{d}\left(V_{+}\right) Q \mathbf{1}=f^{d}\left(V_{-}\right) .
$$

We assume that $w_{ \pm}$define a strong detonation with Lax $n$-shock structure.

Definition 1.1. Let $a^{j}=d f^{j} \in \mathbb{R}^{n \times n}$, and set $\mathcal{A}^{j}=\left(a^{0}\right)^{-1} a^{j}$. The states $w_{ \pm}$are a strong detonation with Lax $n$-shock structure provided:

(a) they satisfy the jump condition (1.2), and

(b) the $n \times n$ matrix $\mathcal{A}^{d}\left(V_{+}\right)$has $n$ eigenvalues $<0$, while $\mathcal{A}^{d}\left(V_{-}\right)$has $n-1$ eigenvalues $<0$ and one eigenvalue $>0$.

Remark 1.1. 1. The existence of CJ solutions as in Definition 1.1 for the physical equations is proved in [CF, FD]. We assume their existence for the abstract model.

2. The case of a planar front moving with nonzero constant velocity can be reduced to the present case by a change of frame. 
3. In the case of the physical equations, say when $d=3$, the eigenvalues of $\mathcal{A}^{d}(V)$ are (recall $V=(\rho, \mathbf{u}, T)) u_{d}, u_{d}, u_{d}, u_{d} \pm c$, where $c$ is sound speed

$$
c^{2}=p_{\rho}+\frac{p p_{e}}{\rho^{2}}=p_{\rho}+\frac{p p_{T}}{\rho^{2} c_{v}} .
$$

Thus, the assumption of $n$-shock structure corresponds to the statement that unburnt gas is moving from right to left across the front $\left(u_{d+}<0\right)$, and that the gas speed is supersonic ahead of the front $\left(\left|u_{d_{+}}\right|>c_{+}\right)$and subsonic behind $\left(\left|u_{d_{-}}\right|<c_{-}\right)$.

1.2. RNS profile. The RNS profile is a smooth solution $\bar{w}(x)=(\bar{V}(x), \bar{Y}(x))$ of the abstract model (0.22) such that

$$
\bar{w}(x) \rightarrow w_{ \pm} \text {as } x \rightarrow \pm \infty,
$$

where $w_{ \pm}$are given by (1.1). This is equivalent to saying that $\bar{w}(x)$ defines a heteroclinic orbit of the steady travelling-wave ODE:

$$
F^{d}(\bar{w})^{\prime}=\left(B^{d d}(\bar{w}) \bar{w}^{\prime}\right)^{\prime}+R(\bar{w})
$$

connecting the endstates $w_{ \pm}$(the prime denotes differentiation with respect to $x$ ). Writing out equation (1.5)

$$
\begin{aligned}
& (a) f^{d}(\bar{V})^{\prime}=\left(\mathcal{B}^{d d}(\bar{V}) \bar{V}^{\prime}\right)^{\prime}+Q K \psi(\bar{V}) \bar{Y} \\
& (b)\left(g^{d}(\bar{V}) \bar{Y}\right)^{\prime}=\left(D^{d d}(\bar{V}) \bar{Y}^{\prime}\right)^{\prime}-K \psi(\bar{V}) \bar{Y},
\end{aligned}
$$

using (1.6)(b) in (1.6)(a), and integrating $\int_{-\infty}^{x}$ gives

$$
\mathcal{B}^{d d}(\bar{V}) \bar{V}^{\prime}=f^{d}(\bar{V})-f^{d}\left(V_{-}\right)-Q\left(D^{d d}(\bar{V}) \bar{Y}^{\prime}-g^{d}(\bar{V}) \bar{Y}\right)
$$

(recall $\left(Y_{-}=\mathbf{0}\right)$. Clearly, $w_{-}$is a rest point of (1.7). The condition that $w_{+}$be a rest point is the same as the jump condition for the CJ system (1.2).

1.3. ZND profile. The ZND profile $\tilde{w}(x)=(\tilde{V}(x), \tilde{Y}(x))$ is a weak solution of the ZND abstract model $\left((0.22)\right.$ with all $\left.B^{j k}=0\right)$ which satisfies

$$
\begin{aligned}
& \tilde{w}(x)=w_{+} \text {in } x>0 \\
& F^{d}(\tilde{w})^{\prime}=R(\tilde{w}) \text { in } x<0, \tilde{w} \rightarrow w_{-} \text {as } x \rightarrow-\infty
\end{aligned}
$$

and the jump condition at $x=0$

$$
\left[F^{d}(\tilde{w})\right]_{*}=0 .
$$

Here we let $w_{*}=\left(V_{*}, Y_{*}\right)$ denote the von Neumann state $\tilde{w}\left(0^{-}\right)$just to the left of the discontinuity, and

$$
\left[F^{d}(\tilde{w})\right]_{*}=F^{d}\left(w_{+}\right)-F^{d}\left(w_{*}\right) .
$$

Let's do a consistency check. Integrating $\int_{-\infty}^{0}$ as in (1.7) we get

$$
0=f^{d}\left(V_{*}\right)-f^{d}\left(V_{-}\right)+Q\left(g^{d}\left(V_{*}\right) Y_{*}\right) .
$$

Together with the ZND jump condition (1.9), this implies the CJ jump condition (1.2). 
Remark 1.2. 1. In the case of the physical RNS or ZND equations we suppose that the $T$ components of $w_{ \pm}$satisfy

$$
T_{+}<T_{i}, \quad T_{-}>T_{0}
$$

for temperatures $T_{i}, T_{0}$ as in (0.19).

2. RNS profiles are constructed in Gasser-Szmolyan [GS] for the physical equations using geometric singular perturbation theory. Assuming that the dissipative coefficients satisfy

$$
\nu=\delta \hat{\nu}, \epsilon=\delta \hat{\epsilon}, \kappa=\delta \hat{\kappa}, \beta=\delta \hat{\beta}
$$

then for $\delta$ sufficiently small, strong detonation profiles exist and are unique. Moreover, for any fixed $\alpha>0$ they converge as $\delta \rightarrow 0$ to ZND profiles uniformly in $C^{1}(\mathbb{R} \backslash(-\alpha, \alpha)$ ). (As usual, we suppress the dependence of RNS profiles on viscosity in the notation.) See also Gardner [G], Wagner [Wa], and Hesaraaki \& Razani [HR] for other treatments of RNS profiles.

3. In the case of the physical RNS profiles, the transverse velocity components satisfy

$$
\bar{u}_{j}(x)=\text { constant }_{j}, j=1, \ldots, d-1 .
$$

Changing to a moving frame, we can make all the constants 0 . The normal velocity component satisfies

$$
\bar{u}_{d}(x)<-\theta<0 \text { for all } x \text {, for some fixed positive } \theta \text {. }
$$

Again, this corresponds to unburnt gas moving from right to left across the front.

4. Statements (1.14) and (1.15) hold equally well with the ZND velocity profile $\tilde{\mathbf{u}}$ in place of $\overline{\mathbf{u}}$.

1.4. Curved ZND and Chapman-Jouguet fronts. Perturbations cause planar fronts to curve, and the perturbed solutions are no longer travelling waves in the above sense. Before linearizing about planar fronts, we need to write out the jump conditions for the ZND and CJ systems when the surface of discontinuity is curved.

Suppose the ZND front is a surface $\mathcal{S}$ defined by:

$$
x=X\left(t, y^{\prime}\right), y^{\prime}=\left(y_{1}, \ldots, y_{d-1}\right) .
$$

A ZND solution $w\left(t, y^{\prime}, x\right)$ is discontinuous across $\mathcal{S}$, satisfies the ZND system

$$
\sum_{j=0}^{d} F^{j}(w)_{y_{j}}=R(w)
$$

on each side, and satisfies the jump condition

$$
\sum_{j=0}^{d-1} X_{y_{j}}\left[F^{j}(w)\right]_{*}-\left[F^{d}(w)\right]_{*}=0 \text { on } \mathcal{S} .
$$

(recall: $t=y_{0}, x=y_{d}$ ). The functions $w$ and $X$ are coupled through the jump condition, so the problem defined by $(1.17),(1.18)$ is a free boundary problem for the unknowns $(w, X)$. 
Similarly, if we now let $\mathcal{S}$ denote a CJ front, a $C J$ solution $V\left(t, y^{\prime}, x\right)$ satisfies the CJ system

$$
\sum_{j=0}^{d} \tilde{f}^{j}(V)_{y_{j}}=0
$$

on each side and the jump condition

$$
\sum_{j=0}^{d-1} X_{y_{j}}\left[\tilde{f}^{j}(V)\right]-\left[\tilde{f}^{d}(V)\right]=0 \text { on } \mathcal{S} .
$$

Now we have

$$
\tilde{f}^{j}(V)=\left\{\begin{array}{l}
f^{j}(V), \text { for } x<X\left(t, y^{\prime}\right) \\
f^{j}(V)+g^{j}(V) Q \mathbf{1}, \text { for } x>X\left(t, y^{\prime}\right)
\end{array}\right.
$$

\section{Assumptions}

For convenient reference we collect here all the structural and profile assumptions that apply to the abstract models. We emphasize that the functions $f^{j}, g^{j}, B^{j k}$, and $R$ are fixed once and for all; any function that appears in two different models is the same in both models. Every assumption that applies to a given abstract model is satisfied by the corresponding physical system.

Notation 2.1. 1. For $F^{j}(w)$ as in $(0.23)$, let

$$
A^{j}(w)=d_{w} F^{j}(w)=\left(\begin{array}{cc}
d f^{j}(V) & 0 \\
d_{V} g^{j}(V) Y & g^{j}(V) I_{s \times s}
\end{array}\right) \in \mathbb{R}^{(n+s) \times(n+s)} .
$$

Given a scalar function $h(V)$ and $v \in \mathbb{C}^{n}$, we'll often write

$$
d_{V} h(V) Y v:=\left(d_{V} h(V) \cdot v\right) Y .
$$

2. The matrices $B^{j k} \in \mathbb{R}^{(n+s) \times(n+s)}(0.25)$ may be written

$$
B^{j k}(w)=\left(\begin{array}{cc}
\mathcal{B}^{j k}(V) & 0 \\
0 & D^{j k}(V)
\end{array}\right)=\left(\begin{array}{ccc}
0 & 0 & 0 \\
0 & b^{j k}(V) & 0 \\
0 & 0 & D^{j k}(V)
\end{array}\right)=\left(\begin{array}{cc}
0 & 0 \\
0 & \mathbb{B}^{j k}(V)
\end{array}\right)
$$

where $\mathbb{B}^{j k} \in \mathbb{R}^{(r+s) \times(r+s)}$.

3. Let

$$
d f^{j}(V)=a^{j}(V)=\left(\begin{array}{cc}
a_{11}^{j} & a_{12}^{j} \\
a_{21}^{j} & a_{22}^{j}
\end{array}\right) \in \mathbb{R}^{n \times n},
$$

where $a_{11}^{j} \in \mathbb{R}^{(n-r) \times(n-r)}, a_{22}^{j} \in \mathbb{R}^{r \times r}$. Similarly, set $d \tilde{f}^{j}=\tilde{a}^{j}$ for $\tilde{f}^{j}$ as in (1.21).

4. Set $\bar{A}^{j}=\left(A^{0}\right)^{-1} A^{j}$ and $\bar{B}^{j k}=\left(A^{0}\right)^{-1} B^{j k} \quad$ (see (H1) below).

Assumption 2.1. (HO) The states $w_{ \pm}$define a strong detonation with Lax $n$-shock structure (recall Definition 1.1). The RNS and ZND profiles decay exponentially to their endstates with all derivatives: for some $\delta>0$

$$
\left|(d / d x)^{k}\left(\bar{w}-w_{ \pm}\right)\right| \leq C_{k} e^{-\delta|x|} \text { as } x \rightarrow \pm \infty
$$


and similarly for $\tilde{w}$. In addition, the $\tilde{a}^{j}=d \tilde{f}^{j}$ satisfy

$$
\left(\tilde{a}^{0}\right)^{-1} \tilde{a}^{j}=\left(a^{0}\right)^{-1} a^{j} \text { at } V_{+}, j=0, \ldots, d .
$$

Assumption 2.2. There exists an open set $\mathcal{U} \subset \mathbb{R}^{n+s}$ such that the endstates and profiles satisfy:

$$
w_{ \pm} \in \mathcal{U}, \bar{w}(x) \in \mathcal{U}, \tilde{w}(x) \in \mathcal{U} \text { for all } x
$$

and:

(H1) The functions $F^{j}(w)(0.23), B^{j k}(w)(0.25)$, and $R(w)(0.26)$ are defined and $C^{\infty}$ in $\mathcal{U}$. The matrix $A^{0}(w)$ is invertible in $\mathcal{U}$ and $g^{0}(V)>C>0$ in $\mathcal{U}$. The functions $g^{j}(V)$ (0.23) satisfy the following conditions along the RNS and ZND profiles:

$$
\begin{aligned}
& g^{j}(\bar{V})=0, j=1, \ldots, d-1 ; \quad g^{d}(\bar{V})<-\theta<0 \quad(R N S) \\
& g^{j}(\tilde{V})=0, j=1, \ldots, d-1 ; \quad g^{d}(\tilde{V})<-\theta<0 \quad(Z N D)
\end{aligned}
$$

for some fixed $\theta>0$.

(H2) The constant matrix $K \in \mathbb{R}^{s \times s}(0.26)$ is positive definite; the constant matrix $Q \in$ $\mathbb{R}^{n \times s}(0.26)$ has its first $(n-r)$ rows equal to 0 ; the function $\psi(V)$ in $(0.26)$ satisfies $\psi\left(V_{+}\right)=$ $0, \psi\left(V_{-}\right)=1, d \psi\left(V_{+}\right)=0$.

(H3) (partial parabolicity) Let $\overline{\mathbb{B}}^{j k}$ denote the lower right $(r+s) \times(r+s)$ block of $\bar{B}^{j k}$. There exists a $C>0$ such that for all $w \in \mathcal{U}$ and $\xi \in \mathbb{R}^{d}$ the eigenvalues $\mu$ of $\overline{\mathbb{B}}(w, \xi)=$ $\sum_{j, k=1}^{d} \overline{\mathbb{B}}^{j k}(w) \xi_{j} \xi_{k} \in \mathbb{R}^{(r+s) \times(r+s)}$ satisfy

$$
\Re \mu \geq C|\xi|^{2} .
$$

(H4) The matrix $a^{0} \in \mathbb{R}^{n \times n}$ has the structure

$$
a^{0}=\left(\begin{array}{cc}
a_{11}^{0} & 0 \\
a_{21}^{0} & a_{22}^{0}
\end{array}\right)
$$

in $\mathcal{U}$.

(H5) The matrix $a_{11}^{d} \in \mathbb{R}^{(n-r) \times(n-r)}$ is invertible along the $R N S$ and ZND profiles, and $\left(a_{11}^{0}\right)^{-1} a_{11}^{d}$ is negative definite at both endstates $w_{ \pm}$.

Assumption 2.3. (H6) (hyperbolicity) For all $\xi \in \mathbb{R}^{d} \backslash 0$ the eigenvalues of $\bar{A}\left(w_{ \pm}, \xi\right)=$ $\sum_{j=1}^{d} \bar{A}^{j}\left(w_{ \pm}\right) \xi_{j}$ are real and semisimple with constant multiplicity.

(Hr) (strict dissipativity) Let $\bar{B}\left(w_{ \pm}, \xi\right)=\sum_{j, k=1}^{d} \bar{B}^{j k}\left(w_{ \pm}\right) \xi_{j} \xi_{k}$. There is a $C>0$ such that for all $\xi \in \mathbb{R}^{d}$, the eigenvalues $\lambda$ of $i \bar{A}\left(w_{ \pm}, \xi\right)+\bar{B}\left(w_{ \pm}, \xi\right)+\left(A^{0}\right)^{-1} d_{w} R\left(w_{ \pm}\right)$satisfy

$$
\Re \lambda \geq C \frac{|\xi|^{2}}{1+|\xi|^{2}} .
$$

The analysis of the ZND Evans function requires the following assumption.

Assumption 2.4. (H8) For $v \in \mathbb{C}^{n}$ let $N v=Q d_{V} g^{d}\left(V_{*}\right) v Y_{*} \in \mathbb{C}^{n}$. The matrix $a^{d}\left(V_{*}\right)+N$ is invertible.

Remark 2.1. 1. In (H6) semisimple means that algebraic and geometric multiplicities are equal. 
2. Hypotheses $(\mathrm{H} 0),(\mathrm{H} 1),(\mathrm{H} 2),(\mathrm{H} 4)$, and $(\mathrm{H} 5)$ are straightforward to check for the physical equations. For (H0) see Remark 1.1 and [GS]. Note that the equality in (2.6) holds automatically at $V_{-}$since $a^{j}=\tilde{a}^{j}$ at $V_{-}$. In the case of an $s$-step reaction (2.6) follows from the fact that

$$
\tilde{a}^{j}\left(V_{+}\right)=M a^{j}\left(V_{+}\right) \text {, where } M=\left(\begin{array}{ccccc}
1 & 0 & \cdots & 0 & 0 \\
0 & 1 & \cdots & 0 & 0 \\
0 & 0 & \cdots & 1 & 0 \\
\sum_{j=1}^{s} q_{j} & 0 & \cdots & 0 & 1
\end{array}\right) .
$$

To check (2.8) in (H1), recall Remark 1.2, parts 3 and 4. (H2) holds provided we normalize $\rho_{-}=1$. To check (H5), note $a_{11}^{d}=u_{d}$ and use Remark 1.2 again.

3. Hypotheses (H3), (H6), (H7) also hold for the physical equations, but are not easy to check directly. In Kawashima-Shizuta [KaSh1, KaSh2], the analogous hypotheses are verified for the nonreactive Navier-Stokes equations using a symmetrizer derived from an entropy function. From (H4) we see that $\left(A^{0}\right)^{-1}$ has the structure

$$
\left(\begin{array}{ccc}
\left(a_{11}^{0}\right)^{-1} & 0 & 0 \\
* & \left(a_{22}^{0}\right)^{-1} & 0 \\
* & * & \left(g^{0}\right)^{-1} I_{s \times s}
\end{array}\right)
$$

This, together with the fact that the matrix functions $a^{j}, b^{j k}$ are the same in both the RNS and nonreactive NS systems, allows us to use (0.28) to deduce (H3), (H6), and (H7) for the physical RNS equations $(0.15 \mathrm{a}),(0.15 \mathrm{~b}),(0.20),(0.15 \mathrm{~d})$ from the corresponding properties in the nonreactive case. (Also, see the Appendix for a Kawashima-type symmetrizer for RNS.)

4. The validity of (H8) for the physical equations is discussed in part 5; see (8.8).

Remark 2.2. Hypothesis (H0) and (H1) imply that the matrix $\bar{A}^{d}\left(w_{+}\right)$has $n+s$ eigenvalues $<0$, while $\bar{A}^{d}\left(w_{-}\right)$has one eigenvalue $>0$ and $n+s-1$ eigenvalues $<0$.

\section{LinEARIZATION}

3.1. Reactive Navier-Stokes. The linearization of $(0.22)$ about $\bar{w}(x)$ is

$$
\sum_{j=0}^{d}\left(\mathbb{A}^{j}(\bar{w}) w\right)_{y_{j}}=\sum_{j, k=1}^{d}\left(B^{j k}(\bar{w}) w_{y_{k}}\right)_{y_{j}}+d_{w} R(\bar{w}) w,
$$

where

$$
\begin{aligned}
& \mathbb{A}^{j}(\bar{w}) w:=A^{j}(\bar{w}) w-\left(w \cdot d_{w} B^{j d}(\bar{w})\right) \bar{w}^{\prime} \\
& d_{w} R=\left(\begin{array}{cc}
Q K d_{V} \psi Y & Q K \psi \\
-K d_{V} \psi Y & -K \psi
\end{array}\right) .
\end{aligned}
$$

Next, Laplace transform in $t=y_{0}$ and Fourier transform in $y^{\prime}=\left(y_{1}, \ldots, y_{d-1}\right)$ to obtain:

$$
\begin{aligned}
& \left(B^{d d}(\bar{w}) w^{\prime}\right)^{\prime}=\lambda \mathbb{A}^{0}(\bar{w}) w+\sum_{j=1}^{d-1} i \eta_{j} \mathbb{A}^{j}(\bar{w}) w+\left(\mathbb{A}^{d}(\bar{w}) w\right)^{\prime} \\
& -\sum_{j=1}^{d-1} i \eta_{j} B^{j d}(\bar{w}) w^{\prime}-\sum_{k=1}^{d-1} i \eta_{k}\left(B^{d k}(\bar{w}) w\right)^{\prime}+\sum_{j, k=1}^{d-1} B^{j k}(\bar{w}) \eta_{j} \eta_{k} w-d_{w} R(\bar{w}) w .
\end{aligned}
$$


Here $w^{\prime}=\partial_{x} w$ and we've dropped the hat (indicating transform) on $\hat{w}(x, \lambda, \eta)$. This is the same equation one finds for $n(x, \lambda, \eta)$ when seeking normal mode solutions (0.1) to (3.1).

We will use a conjugation argument to reduce the study of (3.3) to the study of the much simpler limiting systems obtained by letting $x \rightarrow \pm \infty$ in the coefficients of (3.3):

$$
\begin{aligned}
& (R N S)_{ \pm}: \\
& B^{d d} w^{\prime \prime}=\left(A^{d}-\sum_{j=1}^{d-1} i \eta_{j}\left(B^{j d}+B^{d j}\right)\right) w^{\prime} \\
& +\left(\lambda A^{0}+\sum_{j=1}^{d-1} A^{j} i \eta_{j}+\sum_{j, k=1}^{d-1} B^{j k} \eta_{j} \eta_{k}\right) w-d_{w} R w,
\end{aligned}
$$

where the coefficients are evaluated at $w_{ \pm}$in $(R N D)_{ \pm}$. Observe that

$$
d_{w} R\left(w_{+}\right)=\left(\begin{array}{cc}
0 & 0 \\
0 & 0
\end{array}\right) ; \quad d_{w} R\left(w_{-}\right)=\left(\begin{array}{cc}
0 & Q K \\
0 & -K
\end{array}\right)=\left(\begin{array}{ccc}
0 & 0 & 0 \\
0 & 0 & Q_{2} K \\
0 & 0 & -K
\end{array}\right)
$$

for $Q_{2} \in \mathbb{R}^{r \times s}$.

One consequence of the $n$-shock assumption (H0) is that most of our analysis will need to focus on $(\mathrm{RNS})_{-}$. Setting $w=\left(v_{1}, v_{2}, \mathrm{y}\right)$ and writing out the components of $(\mathrm{RNS})_{-}$we obtain:

$$
\begin{aligned}
& 0=a_{11}^{d} v_{1}^{\prime}+a_{12}^{d} v_{2}^{\prime}+\lambda a_{11}^{0} v_{1}+\sum_{j=1}^{d-1} i \eta_{j}\left(a_{11}^{j} v_{1}+a_{12}^{j} v_{2}\right) \\
& b^{d d} v_{2}^{\prime \prime}=a_{21}^{d} v_{1}^{\prime}+a_{22}^{d} v_{2}^{\prime}-\sum_{j=1}^{d-1} i \eta_{j}\left(b^{j d}+b^{d j}\right) v_{2}^{\prime} \\
& +\lambda a_{21}^{0} v_{1}+\lambda a_{22}^{0} v_{2}+\sum_{j=1}^{d-1} i \eta_{j}\left(a_{21}^{j} v_{1}+a_{22}^{j} v_{2}\right)+\sum_{j, k=1}^{d-1} b^{j k} \eta_{j} \eta_{k} v_{2}-Q_{2} K \mathrm{y}, \\
& D^{d d} \mathrm{y}^{\prime \prime}=g^{d} I_{s} \mathrm{y}^{\prime}-\sum_{j=1}^{d-1} i \eta_{j}\left(D^{j d}+D^{d j}\right) \mathrm{y}^{\prime}+\left(\lambda g^{0} I_{s}+\sum_{j, k=1}^{d-1} D^{j k} \eta_{j} \eta_{k}+K\right) \mathrm{y}
\end{aligned}
$$

where $I_{s}$ denotes the $s \times s$ identity matrix.

In order to construct stability determinants, it is more convenient to work with an equivalent first-order system. Setting $U=\left(v_{1}, v_{2}, \mathrm{y}, v_{2}^{\prime}, \mathrm{y}^{\prime}\right)$, solving for $v_{1}^{\prime}$ in the first equation of (3.6), and substituting into the second, we may rewrite (RNS) _ in the equivalent form:

$$
U^{\prime}=G_{-}\left(w_{-}, \lambda, \eta\right) U
$$


where $G_{-}$is an $(n+r+2 s) \times(n+r+2 s)$ matrix

$$
G_{-}=\left(\begin{array}{ccccc}
G_{11} & G_{12} & 0 & G_{14} & 0 \\
0 & 0 & 0 & 1 & 0 \\
0 & 0 & 0 & 0 & 1 \\
G_{41} & G_{42} & G_{43} & G_{44} & 0 \\
0 & 0 & G_{53} & 0 & G_{55}
\end{array}\right)
$$

whose entries can easily be read off from (3.6). We have:

$G_{11}=-\left(a_{11}^{d}\right)^{-1}\left(\lambda a_{11}^{0}+\sum_{j=1}^{d-1} i \eta_{j} a_{11}^{j}\right) ; \quad G_{12}=-\left(a_{11}^{d}\right)^{-1}\left(\sum_{j=1}^{d-1} i \eta_{j} a_{12}^{j}\right) ;$

$G_{14}=-\left(a_{11}^{d}\right)^{-1} a_{12}^{d} ; \quad G_{41}=\left(b^{d d}\right)^{-1}\left(\lambda a_{21}^{0}+\sum_{j=1}^{d-1} i \eta_{j} a_{21}^{j}+a_{21}^{d} G_{11}\right) ;$

$G_{42}=\left(b^{d d}\right)^{-1}\left(\lambda a_{22}^{0}+\sum_{j=1}^{d-1} i \eta_{j} a_{22}^{j}+\sum_{j, k=1}^{d-1} b^{j k} \eta_{j} \eta_{k}++a_{21}^{d} G_{12}\right) ; \quad G_{43}=\left(b^{d d}\right)^{-1}\left(-Q_{2} K\right)$

$G_{44}=\left(b^{d d}\right)^{-1}\left(a_{22}^{d}-\sum_{j=1}^{d-1} i \eta_{j}\left(b^{j d}+b^{d j}\right)+a_{21}^{d} G_{14}\right)$

$G_{53}=\left(D^{d d}\right)^{-1}\left(\lambda g^{0} I_{s}+\sum_{j, k=1}^{d-1} D^{j k} \eta_{j} \eta_{k}+K\right) ; \quad G_{55}=\left(D^{d d}\right)^{-1}\left(g^{d} I_{s}-\sum_{j=1}^{d-1} i \eta_{j}\left(D^{j d}+D^{d j}\right)\right)$.

Similarly, there is a limiting matrix $G_{+}$(see Lemma 5.2) in which the matrix coefficients are evaluated at $w_{+}$.

We can write the original system (3.3) in first-order form

$$
U^{\prime}=G(\bar{w}(x), \lambda, \eta) U
$$

where for some $\delta>0$

$$
\left|G(\bar{w}, \lambda, \eta)-G_{ \pm}\left(w_{ \pm}, \lambda, \eta\right)\right| \leq C e^{-\delta|x|} \text { as } x \rightarrow \pm \infty
$$

uniformly for $|\lambda, \eta|$ in bounded sets.

3.2. ZND. The ZND system (1.17), (1.18) is a free boundary problem for the unknowns $(w, X)$. First we reduce to a fixed boundary problem by changing coordinates

$$
\left(t, y^{\prime}, x^{*}\right)=\left(t, y^{\prime}, x-X\left(t, y^{\prime}\right), w^{*}\left(t, y^{\prime}, x^{*}\right)=w\left(t, y^{\prime}, x\right) .\right.
$$

With stars dropped, the problem in the new coordinates is

$$
\begin{aligned}
& \sum_{j=0}^{d-1} F^{j}(w)_{y_{j}}+\mathcal{F}^{d}(w, d X)_{x}=R(w) \\
& \sum_{j=0}^{d-1} X_{y_{j}}\left[F^{j}(w)\right]_{*}-\left[F^{d}(w)\right]_{*}=0 \text { on } x=0,
\end{aligned}
$$


where

$$
\mathcal{F}^{d}(w, d X)=F^{d}(w)-\sum_{j=0}^{d-1} X_{y_{j}} F^{j}(w) .
$$

The problem (3.13) can be viewed as a transmission problem for unknowns $\left(w_{ \pm}\left(t, y^{\prime}, x\right), X\left(t, y^{\prime}\right)\right)$ in $\pm x \geq 0$, with transmission conditions given by the jump condition on $x=0$. We'll usually suppress the \pm on $w$ (partly to avoid confusion with the endstates $w_{ \pm}$).

Linearizing (3.13) with respect to both $w$ and $X$ about the stationary solution given by the ZND profile $\tilde{w}(x)$ and front $X=0$, we obtain

$$
\begin{aligned}
& \sum_{j=0}^{d-1} A^{j}(\tilde{w})\left(\partial_{y_{j}} w-X_{y_{j}} \tilde{w}^{\prime}\right)+\left(A^{d}(\tilde{w}) w\right)^{\prime}=d_{w} R(\tilde{w}) w \\
& \sum_{j=0}^{d-1} X_{y_{j}}\left[F^{j}(\tilde{w})\right]_{*}-\left[A^{d}(\tilde{w}) w\right]_{*}=0 \text { on } x=0,
\end{aligned}
$$

where $w$ and $X$ now denote perturbations. Fourier-Laplace transformation gives (dropping hats on $w$ and $X$ )

$$
\begin{aligned}
& \lambda A^{0}(\tilde{w})\left(w-X \tilde{w}^{\prime}\right)+\sum_{j=1}^{d-1} i \eta_{j} A^{j}(\tilde{w})\left(w-X \tilde{w}^{\prime}\right)+\left(A^{d}(\tilde{w}) w\right)^{\prime}=d_{w} R(\tilde{w}) w \\
& X\left(\lambda\left[F^{0}(\tilde{w})\right]_{*}+\sum_{j=1}^{d-1} i \eta_{j}\left[F^{j}(\tilde{w})\right]_{*}\right)-\left[A^{d}(\tilde{w}) w\right]_{*}=0 \text { on } x=0 .
\end{aligned}
$$

For the stability analysis it is convenient to eliminate $X$ from the interior equation by defining new unknowns ( \pm suppressed)

$$
w^{\#}=w-X \tilde{w}^{\prime}
$$

This gives the equivalent problem

$$
\begin{aligned}
& \text { (a) }\left(\lambda A^{0}(\tilde{w})+\sum_{j=1}^{d-1} i \eta_{j} A^{j}(\tilde{w})\right) w^{\#}+\left(A^{d}(\tilde{w}) w^{\#}\right)^{\prime}=d_{w} R(\tilde{w}) w^{\#} \\
& \text { (b) } X\left(\lambda\left[F^{0}(\tilde{w})\right]_{*}+\sum_{j=1}^{d-1} i \eta_{j}\left[F^{j}(\tilde{w})\right]_{*}-\left[A^{d}(\tilde{w}) \tilde{w}^{\prime}\right]_{*}\right)-\left[A^{d}(\tilde{w}) w^{\#}\right]_{*}=0 \text { on } x=0 .
\end{aligned}
$$

Here we've used the relation

$$
\left(A^{d}(\tilde{w}) \tilde{w}^{\prime}\right)^{\prime}=d_{w} R(\tilde{w}) \tilde{w}^{\prime},
$$

obtained by differentiating the profile equation in (1.8). In computing jumps involving the derivative of the profile, $\tilde{w}^{\prime}$, we use

$$
\begin{aligned}
& \lim _{x \rightarrow 0^{+}} \tilde{w}^{\prime}=0 \\
& \lim _{x \rightarrow 0^{-}} \tilde{w}^{\prime}=\tilde{w}_{-}^{\prime}(0),
\end{aligned}
$$

as is appropriate for the linearized transmission problem. 
Henceforth, we'll work with the form of the ZND problem given by (3.18), dropping the \# on $w$. The limiting systems obtained by letting $x \rightarrow \pm \infty$ in (3.18)(a) are

$$
(Z N D)_{ \pm} \quad\left(\lambda A^{0}\left(w_{ \pm}\right)+\sum_{j=1}^{d-1} i \eta_{j} A^{j}\left(w_{ \pm}\right)\right) w+A^{d}\left(w_{ \pm}\right) w^{\prime}=d_{w} R\left(w_{ \pm}\right) w .
$$

As with RNS our analysis will focus mainly on the minus side. With $w=(v, \mathrm{y})$ we can write $(3.21)$ as the equivalent $(n+s) \times(n+s)$ system

$$
w^{\prime}=\mathcal{G}_{-}\left(w_{-}, \lambda, \eta\right) w
$$

where

$$
\begin{aligned}
& \mathcal{G}_{-}=\left(\begin{array}{cc}
H_{-}(\lambda, \eta) & \left(a^{d}\right)^{-1} Q K \\
0 & -\left(g^{d}\right)^{-1}\left(\lambda g^{0} I_{s}+K\right)
\end{array}\right) \text { with } \\
& H_{ \pm}(\lambda, \eta)=-\left(a^{d}\right)^{-1}\left(\lambda a^{0}+\sum_{j=1}^{d-1} i \eta_{j} a^{j}\right)\left(V_{ \pm}\right) .
\end{aligned}
$$

The matrix $\mathcal{G}_{+}\left(w_{+}, \lambda, \eta\right)$ is defined similarly (see (4.10).

Similarly, we can write the variable-coefficient system (3.18)(a) in the form

$$
w^{\prime}=\mathcal{G}(\tilde{w}(x), \lambda, \eta) w \text { in } \pm x>0
$$

where for some $\delta>0$

$$
\left|\mathcal{G}(\tilde{w}, \lambda, \eta)-\mathcal{G}_{-}\left(w_{-}, \lambda, \eta\right)\right| \leq C e^{-\delta|x|} \text { as } x \rightarrow-\infty
$$

uniformly for $|\lambda, \eta|$ in bounded sets.

3.3. Chapman-Jouget. The process in this case parallels that for ZND. After the same change of variables (3.12), the CJ problem (1.19), (1.20) takes the form

$$
\begin{aligned}
& \sum_{j=0}^{d-1} \tilde{f}^{j}(V)_{y_{j}}+\tilde{\mathbf{f}}^{d}(V, d X)_{x}=0 \\
& \sum_{j=0}^{d-1} X_{y_{j}}\left[\tilde{f}^{j}(V)\right]-\left[\tilde{f}^{d}(V)\right]=0 \text { on } x=0,
\end{aligned}
$$

where

$$
\tilde{\mathbf{f}}^{d}(V, d X)=\tilde{f}^{d}(V)-\sum_{j=0}^{d-1} X_{y_{j}} \tilde{f}^{j}(V)
$$

Linearizing with respect to both $V$ and $X$ about the stationary solution given by $V_{ \pm}$and the front $X=0$, and taking the Laplace-Fourier transform as before, we get the transmission 
problem

$$
\begin{aligned}
& \text { (a) } \lambda \tilde{a}^{0} v+\sum_{j=1}^{d-1} i \eta_{j} \tilde{a}^{j} v+\tilde{a}^{d} v^{\prime}=0 \\
& \text { (b) } X\left(\lambda\left[\tilde{f}^{0}\right]+\sum_{j=1}^{d-1} i \eta_{j}\left[\tilde{f}^{j}\right]\right)-\left[\tilde{a}^{d} v\right]=0 \text { on } x=0,
\end{aligned}
$$

where $(v, X)$ now denotes the (transformed) perturbation, $\tilde{a}^{j}:=d \tilde{f}^{j}$, and the $\tilde{f}^{j}$, $\tilde{a}^{j}$ are evaluated at $V_{ \pm}$in $\pm x>0$. The interior problem (3.28)(a) can be rewritten

$$
\begin{aligned}
& v^{\prime}=\tilde{H}_{ \pm}(\lambda, \eta) v \text { in } \pm x>0, \text { with } \\
& \tilde{H}_{ \pm}(\lambda, \eta)=-\left(\tilde{a}^{d}\right)^{-1}\left(\lambda \tilde{a}^{0}+\sum_{j=1}^{d-1} i \eta_{j} \tilde{a}^{j}\right)\left(V_{ \pm}\right) .
\end{aligned}
$$

Note that because of Hypothesis $(\mathrm{H} 0)$, we have $\tilde{H}_{ \pm}=H_{ \pm}$for $H_{ \pm}$as in (3.23).

\section{Stability Determinants}

4.1. The Chapman-Jouguet determinant. First we define the determinant $\Delta_{C J}(\lambda, \eta)$, whose zeros in $\Re \lambda>0$ correspond to solutions of the linearized CJ problem that grow exponentially with time.

For $\gamma=\Re \lambda>0$ let $F_{ \pm}(\zeta)$ (recall $\zeta=(\tau, \gamma, \eta)=\rho \hat{\zeta}$ ) be the generalized eigenspace of $H_{ \pm}(\zeta)$ corresponding to eigenvalues with negative (resp. positive) real part. The hyperbolicity hypothesis (H6) implies that the dimensions of $F_{ \pm}(\zeta)$ are constant in $\gamma>0$, so we can set $(\tau, \eta)=0$ in (3.29) and use the assumption of $n$-shock structure (H0) to see that

$$
\operatorname{dim} F_{+}(\zeta)=0 ; \operatorname{dim} F_{-}(\zeta)=n-1 \text { in } \gamma>0 .
$$

A classical argument based on conjugation of $H_{-}(\zeta)$ to block structure shows that $F_{-}(\zeta)$ is $C^{\infty}$ in $\gamma>0$ and extends continuously to $\gamma \geq 0$ in $\{|\zeta| \neq 0\}$ (see $[\mathrm{K}]$ or $[\mathrm{CP}]$, chapter 7 ). So we may choose a basis

$$
s_{-}^{j}(\zeta), j=1, \ldots, n-1
$$

for $F_{-}(\zeta)$, locally near any point $\zeta^{*} \neq 0$, where the $s_{-}^{j}$ are homogeneous of degree 0 for $\zeta \neq 0$ and $C^{\infty}$ in $\gamma>0$ with continuous extensions to $\gamma \geq 0$ (in fact the $s_{-}^{j}$ extend smoothly away from glancing points).

Remark 4.1. Consider the functions $s_{-}^{j}(\hat{\zeta})$ on $S_{+}^{d}$, which have been been defined only in a neighborhood of some fixed, arbitrary basepoint $\hat{\zeta}^{*} \in S_{+}^{d}$. Generally, the individual $s_{-}^{j}$ do not extend to functions on all of $S_{+}^{d}$ with the above regularity. However, since the spaces $F_{-}(\hat{\zeta})$ do possess this regularity globally, we can choose the $s_{-}^{j}$ in a family of neighborhoods covering the sphere so that the wedge products

$$
s_{-}^{1}(\hat{\zeta}) \wedge s_{-}^{2}(\hat{\zeta}) \wedge \cdots \wedge s_{-}^{n-1}(\hat{\zeta})
$$

have the same regularity globally on $S_{+}^{d}$. We use this observation below to obtain globally regular stability determinants. 
Inspection of (3.28), (3.29) shows that the linearized CJ problem has solutions growing exponentially with time if for $\gamma>0$ the $n$ vectors

$$
\lambda\left[\tilde{f}^{0}\right]+\sum_{j=1}^{d-1} i \eta_{j}\left[\tilde{f}^{j}\right], \quad r_{-}^{j}(\zeta):=a^{d}\left(V_{-}\right) s_{-}^{j}(\zeta), j=1, \ldots, n-1
$$

are linearly dependent (recall $\left.\tilde{a}^{j}\left(V_{-}\right)=a^{j}\left(V_{-}\right)\right)$.

In view of $(2.8)$ we have

$$
\left[\tilde{f}^{0}\right]=\left[f^{0}\right]+g^{0}\left(V_{+}\right) Q \mathbf{1},\left[\tilde{f}^{j}\right]=\left[f^{j}\right], j=1, \ldots, d-1,
$$

so linear dependence of the vectors (4.4) is equivalent to vanishing of the determinant

$$
\Delta_{C J}(\zeta)=\operatorname{det}\left(r_{-}^{1}(\zeta), \ldots, r_{-}^{n-1}(\zeta), \lambda\left[f^{0}\right]+\sum_{j=1}^{d-1} i \eta_{j}\left[f^{j}\right]+\lambda g^{0}\left(V_{+}\right) Q \mathbf{1}\right)
$$

Remark 4.2. In view of Remark 4.1, if we identify the determinant in (4.6) with the wedge product

$$
r_{-}^{1}(\zeta) \wedge r_{-}^{2}(\zeta) \wedge \cdots \wedge r_{-}^{n-1}(\zeta) \wedge\left(\lambda\left[f^{0}\right]+\sum_{j=1}^{d-1} i \eta_{j}\left[f^{j}\right]+\lambda g^{0}\left(V_{+}\right) Q \mathbf{1}\right)
$$

we obtain a function $\Delta_{C J}$ that is $C^{\infty}$ in $\gamma>0$, continuous in $\{|\zeta| \neq 0, \gamma \geq 0\}$, and satisfies

$$
\Delta_{C J}(\hat{\zeta}, \rho)=\rho \Delta_{C J}(\hat{\zeta}, 1) .
$$

When $Q=0$ this coincides with the Majda determinant for a Lax $n$-shock [Ma1].

4.2. The ZND Evans function. Consider the linearized, transformed ZND problem (3.24)

$$
w^{\prime}=\mathcal{G}(\tilde{w}(x), \zeta) w \text { in } \pm x>0 .
$$

Let $\hat{\zeta}^{*}$ denote an arbitrary fixed basepoint in $S_{+}^{d}$. The first step in constructing the ZND Evans function for low frequencies is to find, locally near $(\hat{\zeta}, \rho)=\left(\hat{\zeta}^{*}, 0\right)$, a basis for the solutions of (4.9) that decay to zero as $x \rightarrow \pm \infty$ for $\hat{\gamma}>0, \rho>0$. The exponential decay of the ZND profile to its endstates (2.5) will allow us obtain such a basis by considering the limiting problems defined by the matrices

$$
\begin{aligned}
& \mathcal{G}_{-}=\left(\begin{array}{cc}
H_{-}(\lambda, \eta) & \left(a^{d}\right)^{-1} Q K \\
0 & -\left(g^{d}\right)^{-1}\left(\lambda g^{0} I_{s}+K\right)
\end{array}\right) \\
& \mathcal{G}_{+}=\left(\begin{array}{cc}
H_{+}(\lambda, \eta) & 0 \\
G_{21} & -\left(g^{d}\right)^{-1} \lambda g^{0} I_{s}
\end{array}\right),
\end{aligned}
$$

where the coefficients are evaluated at $V_{-}$(resp. $\left.V_{+}\right)$.

We first conjugate the variable coefficient problem (4.9) in $x<0$ to the constant coefficient problem

$$
\omega^{\prime}=\mathcal{G}_{-}\left(w_{-}, \lambda, \eta\right) \omega \text { in } x<0,
$$

using the following result, whose proof is established in Lemma 2.6 of [MZ1]: 
Lemma 4.1. Fix a basepoint $P^{*}=\left(\lambda^{*}, \eta^{*}\right)$ in $\Re \lambda \geq 0$. There exists a $C^{\infty},(n+s) \times(n+s)$ matrix $Z_{-}(x, \zeta)$, defined for all $x \leq 0$ and for $\zeta$ in a neighborhood of $P^{*}$, such that

(a) $\partial_{x} Z_{-}=\mathcal{G}(\tilde{w}, \zeta) Z_{-}-Z_{-} \mathcal{G}\left(w_{-}, \zeta\right)$ in $x \leq 0$

(b) $\left|Z_{-}^{-1}\right| \leq C$

(c) $\left|\partial_{x}^{k} \partial_{\zeta}^{\alpha}\left(Z_{-}-I\right)\right| \leq C_{k, \alpha} e^{-\delta|x|}$,

for positive constants $C, \delta$ independent of $(x, \zeta)$ as above.

Observe that because of $(4.12)(\mathrm{a}), w(x)$ is a solution of (4.9) if and only if $\omega(x)$ defined by

$$
w=Z_{-}(x, \zeta) \omega
$$

is a solution of (4.11). The extra properties (4.12)(b),(c) imply that $Z_{-}$establishes a very useful correspondence between solutions of the two problems. In this paper the case where the basepoint $P^{*}=(0,0)$ is especially important.

Remark 4.3. Near a basepoint for which $\gamma^{*}>0$, separation of the generalized eigenspaces of $\mathcal{G}_{-}$corresponding to eigenvalues with positive and negative real parts allows one to prove Lemma 4.1 using classical results in asymptotic ODE theory [Co]. However, since $\mathcal{G}_{-}$has a multiple zero eigenvalue at $\rho=0$, those results don't apply near $\rho=0$ and a more recent tool, the Gap Lemma of [GZ, KS], is needed in their place ([MZ1], Lemma 2.6).

It is helpful to perform a further conjugation as follows. Let

$$
\tilde{K}(\lambda)=-\left(g^{d}\right)^{-1}\left(\lambda g^{0} I_{s}+K\right),
$$

the lower right entry of $\mathcal{G}_{-}$. The eigenvalues $\mu_{j}(\lambda)$ of $\tilde{K}(\lambda)$ satisfy

$$
\Re \mu_{j}(\lambda) \geq C>0 .
$$

Lemma 4.2. For $\rho=|\zeta|$ small, there exists a $C^{\infty}$ matrix

$$
T(\zeta)=\left(\begin{array}{cc}
1 & t_{12}(\zeta) \\
0 & 1
\end{array}\right)
$$

such that

$$
T^{-1}(\zeta) \mathcal{G}_{-}\left(w_{-}, \zeta\right) T(\zeta)=\left(\begin{array}{cc}
H_{-}(\zeta) & 0 \\
0 & \tilde{K}(\lambda)
\end{array}\right):=\mathcal{G}_{D}(\zeta)
$$

The $n \times$ s entry $t_{12}(\zeta)$ is $O(1)$ for $\rho$ small.

Proof. One can posit $T$ of the given form, equate entries in the equation $\mathcal{G}_{-} T=T \mathcal{G}_{D}$, and use the invertibility of $K$ and the smallness of $\rho$ to solve for the $t_{12}$ entry.

We can now use the conjugators $T$ and $Z_{-}$to construct a basis for the space of decaying solutions of $(4.9)$ in $x<0$ when $\hat{\gamma}>0$ and $(\hat{\zeta}, \rho)$ is near $\left(\hat{\zeta}^{*}, 0\right)$. Writing $H_{-}(\zeta)=\rho H_{-}(\hat{\zeta})$ and using the basis vectors $s_{-}^{j}(\hat{\zeta})(4.2)$ for the positive generalized eigenspace space of $H_{-}(\hat{\zeta})$, we see that there are $n-1$ slow modes given by

$$
w^{j}(x, \hat{\zeta}, \rho)=\left(\begin{array}{c}
v^{j} \\
\mathbf{y}^{j}
\end{array}\right)=Z_{-}(x, \zeta) T(\zeta)\left(\begin{array}{c}
e^{x \rho H_{-}(\hat{\zeta})} s_{-}^{j}(\hat{\zeta}) \\
0
\end{array}\right), j=1, \ldots, n-1
$$


and $s$ fast modes given by

$$
w^{j}(x, \zeta)=Z_{-}(x, \zeta) T(\zeta)\left(\begin{array}{c}
0 \\
e^{x \tilde{K}(\lambda)} t_{j}
\end{array}\right), j=n, \ldots, n-1+s,
$$

where $\left\{t_{j}, j=n, \ldots, n-1+s\right\}$ is any basis of $\mathbb{C}^{s}$.

Remark 4.4. By inspection using the known regularity of the factors in (4.18), (4.19), we see that for all $j$ and $\rho$ small, $w^{j}(x, \hat{\zeta}, \rho)$ is defined and $C^{\infty}$ in $\hat{\gamma}>0, \rho \geq 0$, for $\hat{\zeta}$ near $\hat{\zeta}^{*} \in S_{+}^{d}$. For $j=1, \ldots, n-1, w^{j}$ extends continuously to $\hat{\gamma} \geq 0, \rho \geq 0$; for $j=n, \ldots, n-1+s$, there is a $C^{\infty}$ extension.

Similarly, one can construct a conjugator $Z_{+}$which intertwines solutions of $(4.9)$ in $x>0$ with solutions of the problem defined by $\mathcal{G}_{+}$. It follows directly from $(4.1)$ and $-\left(g^{d}\right)^{-1} g^{0}>0$ that, for $\hat{\gamma}>0, \rho>0$, the only solution of

$$
\omega^{\prime}=\mathcal{G}_{+}\left(w_{+}, \lambda, \eta\right) \omega \text { in } x>0
$$

decaying to 0 as $x \rightarrow+\infty$ is the trivial solution.

Defining $w_{-}^{j}=w^{j}$ as above and setting $w_{+}^{j}=0$, we obtain a basis for the space of decaying solutions $\left(w_{+}, w_{-}\right)$of (4.9). In view of the equivalence of the linearized problems (3.18)(a) and (3.24), we see that the transmission problem (3.18) has solutions growing exponentially in time if and only if for some $\gamma>0$ the $n+s$ vectors

$$
A^{d}\left(w_{*}\right) w^{1}\left(0^{-}\right), \ldots, A^{d}\left(w_{*}\right) w^{n-1+s}\left(0^{-}\right), \lambda\left[F^{0}(\tilde{w})\right]_{*}+\sum_{j=1}^{d-1} i \eta_{j}\left[F^{j}(\tilde{w})\right]_{*}+A^{d}\left(w_{*}\right) \tilde{w}^{\prime}\left(0^{-}\right)
$$

are linearly dependent $\left(w_{*}=\left(V_{*}, Y_{*}\right)\right.$ is the von Neumann state just to the left of the front).

For $v \in \mathbb{C}^{n}$ let

$$
S v=d_{V} g^{d}\left(V_{*}\right) v Y_{*} \in \mathbb{C}^{s}
$$

and set

$$
\begin{aligned}
& f^{\eta}=\sum_{j=1}^{d-1} \eta_{j} f^{j} \\
& \mathcal{Z}(\zeta)=-S\left(a^{d}\left(V_{*}\right)^{-1}\left(\lambda\left[f^{0}(\tilde{V})\right]_{*}+i\left[f^{\eta}(\tilde{V})\right]_{*}\right)\right) .
\end{aligned}
$$

Recalling the definition of $A^{d}$ and $F^{j}$, we obtain that the vectors (4.21) are linearly dependent if and only if the Evans determinant

$$
\begin{aligned}
& D_{Z N D}(\hat{\zeta}, \rho)= \\
& \left.\operatorname{det}\left(\begin{array}{cccc}
a^{d}\left(V_{*}\right) v^{1} & \ldots & a^{d}\left(V_{*}\right) v^{n-1+s} & \lambda\left[f^{0}(\tilde{V})\right]_{*}+i\left[f^{\eta}(\tilde{V})\right]_{*}+a^{d}\left(V_{*}\right) \tilde{V}^{\prime} \\
S v^{1}+g^{d}\left(V_{*}\right) \mathrm{y}^{1} & \ldots & S v^{n-1+s}+g^{d}\left(V_{*}\right) \mathrm{y}^{n-1+s} & \lambda\left[g^{0}(\tilde{V}) \tilde{Y}\right]_{*}+S \tilde{V}^{\prime}+g^{d}\left(V_{*}\right) \tilde{Y}^{\prime}
\end{array}\right)\right|_{x=0^{-}}
\end{aligned}
$$


vanishes. After an obvious row operation, this simplifies to

$$
\begin{aligned}
& D_{Z N D}(\hat{\zeta}, \rho)= \\
& \left.\operatorname{det}\left(\begin{array}{cccc}
a^{d}\left(V_{*}\right) v^{1} & \cdots & a^{d}\left(V_{*}\right) v^{n-1+s} & \lambda\left[f^{0}(\tilde{V})\right]_{*}+i\left[f^{\eta}(\tilde{V})\right]_{*}+a^{d}\left(V_{*}\right) \tilde{V}^{\prime} \\
g^{d}\left(V_{*}\right) \mathrm{y}^{1} & \cdots & g^{d}\left(V_{*}\right) \mathrm{y}^{n-1+s} & \lambda\left[g^{0}(\tilde{V}) \tilde{Y}\right]_{*}+\mathcal{Z}(\zeta)+g^{d}\left(V_{*}\right) \tilde{Y}^{\prime}
\end{array}\right)\right|_{x=0^{-}} .
\end{aligned}
$$

$D_{Z N D}$ has been defined for $\rho$ small and $\hat{\zeta}$ near any $\zeta^{*} \in S_{+}^{d}$, with the regularity on $\hat{\gamma} \geq 0$, $\rho \geq 0$ implied by Remark (4.4).

Remark 4.5. 1. Using wedge products as in Remarks 4.1 and 4.2 and recalling Remark 4.4, we obtain a function $D_{Z N D}(\hat{\zeta}, \rho)$ defined on $S_{+}^{d} \times\left[0, \rho_{0}\right]$ for $\rho_{0}$ small that is $C^{\infty}$ in $(\hat{\zeta}, \rho)$ for $\hat{\gamma}>0, \rho \geq 0$, with a continuous extension to $\hat{\gamma} \geq 0, \rho \geq 0$. The argument uses simple connectedness, the fact that the above $C^{\infty}$ regularity is actually real analyticity, and analytic continuation. For more detail see [Z], p.325. The analysis in section 5 will show that the same is true for $D_{Z N D} / \rho$.

2. It is clear from (4.10) that when $\hat{\gamma}>0, \rho>0$ the matrix $\mathcal{G}_{-}$has no pure imaginary eigenvalues. Thus, we can define $D_{Z N D}$ locally near any basepoint $P^{*}$ in $\hat{\gamma}>0, \rho>0$ by the above formula (4.25), except that we now replace the modes $w^{j}$ used there by

$$
w^{j}(x, \zeta)=Z_{-}(x, \zeta) \omega^{j}(x, \zeta), j=1, \ldots, n-1,
$$

where the $\omega^{j}$ are (fast) decaying solutions to the limiting problem in $x \leq 0$ defined for $\zeta$ near $P^{*}$. Using wedge products and analytic continuation as above, $D_{Z N D}$ can be extended real analytically to all of $\hat{\gamma}>0, \rho>0$. The behavior near $\hat{\gamma}=0$ for $\rho$ bounded away from zero is more subtle, and will not be discussed here. Note, for example, that the proof of Lemma 4.2 breaks down in this frequency domain.

The following propositions play an important role in the proof of the Equivalence Theorem.

Proposition 4.1. Slow modes satisfy

$$
\lim _{x \rightarrow-\infty} w^{j}(x, \hat{\zeta}, 0)=\left(\begin{array}{c}
s_{-}^{j}(\hat{\zeta}) \\
0
\end{array}\right)
$$

while fast modes satisfy

$$
\lim _{x \rightarrow-\infty} w^{j}(x, 0)=\left(\begin{array}{l}
0 \\
0
\end{array}\right)
$$

Proof. The result follows by inspection of the formulas (4.18), (4.19), using the properties of $Z_{-}$and $T$.

It is important to make a careful choice of one of the fast modes. The ZND profile equation implies that $\tilde{w}^{\prime}(x)$ is a fast decaying solution of

$$
w^{\prime}=\mathcal{G}(\tilde{w}(x), 0) w \text { in } x<0
$$

(recall $(3.19))$. The next Proposition shows $\tilde{w}^{\prime}(x)$ can be extended smoothly to nonzero frequencies as a fast decaying solution of (4.9).

Proposition 4.2. There exists a $\underline{t} \in \mathbb{C}^{s}$ such that the fast mode given by 


$$
\underline{w}(x, \zeta)=Z_{-}(x, \zeta) T(\zeta)\left(\begin{array}{c}
0 \\
e^{x \tilde{K}(\lambda)} \underline{t}
\end{array}\right)
$$

in $x<0$ is $C^{\infty}$ and satisfies

$$
\underline{w}(x, 0)=\tilde{w}^{\prime}(x) .
$$

Proof. Since $\tilde{w}^{\prime}(x)$ is a solution of (4.29), the given properties of $Z_{-}$and $T$ imply that

$$
\tilde{w}^{\prime}(x)=Z_{-}(x, 0) T(0)\left(\begin{array}{c}
0 \\
e^{x K} \underline{t}
\end{array}\right)
$$

for some $\underline{t} \in \mathbb{C}^{s}$. Inserting this $\underline{t}$ in (4.30) gives the desired fast mode.

Henceforth, we shall take this mode to be the $(n-1+s)$-th fast mode:

$$
w^{n-1+s}(x, \zeta)=\underline{w}(x, \zeta) .
$$

4.3. The RNS Evans function. To construct the RNS Evans function we first conjugate the $(n+r+2 s) \times(n+r+2 s)$ linearized problem (3.10)

$$
U^{\prime}=G(\bar{w}(x), \zeta) U \text { on } \mathbb{R}_{x}
$$

to constant-coefficient limiting problems on $\pm x \geq 0$. For this we use the following lemma, which is proved using the exponential decay (3.11) just like Lemma 4.1.

Lemma 4.3. Fix a basepoint $P^{*}$ in $\Re \lambda \geq 0$. There exist $C^{\infty},(n+r+2 s) \times(n+r+2 s)$ matrices $Z_{ \pm}(x, \zeta)$, defined for $\pm x \geq 0$ and for $\zeta$ in a neighborhood of $P^{*}$, such that

(a) $\partial_{x} Z_{ \pm}=G(\bar{w}, \zeta) Z_{ \pm}-Z_{ \pm} G_{ \pm}\left(w_{ \pm}, \zeta\right)$ in $\pm x \geq 0$

(b) $\left|Z_{ \pm}^{-1}\right| \leq C$

(c) $\left|Z_{ \pm}-I\right| \leq C e^{-\delta|x|}$,

for positive constants $C, \delta$ independent of $(x, \zeta)$ as above. Here $G_{-}$is defined in (3.8), (3.9) and $G_{+}$is given by

$$
G_{+}=\left(\begin{array}{ccccc}
\mathcal{G}_{11} & \mathcal{G}_{12} & 0 & \mathcal{G}_{14} & 0 \\
0 & 0 & 0 & 1 & 0 \\
0 & 0 & 0 & 0 & 1 \\
\mathcal{G}_{41} & \mathcal{G}_{42} & 0 & \mathcal{G}_{44} & 0 \\
\mathcal{G}_{51} & \mathcal{G}_{52} & \mathcal{G}_{53} & \mathcal{G}_{54} & \mathcal{G}_{55}
\end{array}\right)
$$

where now

$$
\begin{aligned}
& \mathcal{G}_{51}=\left(D^{d d}\right)^{-1}\left(\lambda \partial_{v_{1}} g^{0} Y_{+}+\sum_{j=1}^{d-1} i \eta_{j} \partial_{v_{1}} g^{j} Y_{+}+\partial_{v_{1}} g^{d} Y_{+} \mathcal{G}_{11}\right) \\
& \mathcal{G}_{52}=\left(D^{d d}\right)^{-1}\left(\lambda \partial_{v_{2}} g^{0} Y_{+}+\sum_{j=1}^{d-1} i \eta_{j} \partial_{v_{2}} g^{j} Y_{+}+\partial_{v_{1}} g^{d} Y_{+} \mathcal{G}_{12}\right) \\
& \mathcal{G}_{54}=\left(D^{d d}\right)^{-1}\left(\partial_{v_{2}} g^{d} Y_{+}+\partial_{v_{1}} g^{d} Y_{+} \mathcal{G}_{14}\right) .
\end{aligned}
$$


The remaining nonzero entries are given by the same formulas as the corresponding entries in (3.9), except that now all matrix coefficients are evaluated at $w_{+}$, and $K$ should be set equal to zero.

Proposition 4.3. For $\zeta \neq 0$ and $\gamma \geq 0$, the matrices $G_{ \pm}\left(w_{ \pm}, \zeta\right)$ have no purely imaginary eigenvalues.

Proof. Suppose $\mu=i \xi_{d}, \xi_{d} \in \mathbb{R}$, is an eigenvalue of $G_{ \pm}\left(w_{ \pm}, \zeta\right)$, where $\zeta=(\tau, \gamma, \eta) \neq 0$. Letting $\xi=\left(\eta, \xi_{d}\right)$ and tracing back through the definitions, this implies that $-\lambda=-i \tau-\gamma$ is an eigenvalue of

$$
i \bar{A}\left(w_{ \pm}, \xi\right)+\bar{B}\left(w_{ \pm}, \xi\right)-\left(A^{0}\left(w_{ \pm}\right)\right)^{-1} d_{w} R\left(w_{ \pm}\right)
$$

Strict dissipativity (H7) implies that

$$
-\gamma \geq C \frac{|\xi|^{2}}{1+|\xi|^{2}}
$$

Thus, $\gamma=0, \xi=0$, and hence, because of the positivity of $K, \tau=0$; so $\zeta=0$, a contradiction.

Corollary 4.1. The number (counted with multiplicities) of eigenvalues $\mu$ of $G_{-}\left(w_{-}, \zeta\right)$ in $\Re \mu>0$ and in $\Re \mu<0$ is independent of $\zeta$ for $\zeta \neq 0$ and $\gamma \geq 0$. The same applies to $G_{+}\left(w_{+}, \zeta\right)$.

Next we perform a further conjugation of $G_{ \pm}$to a block diagonal form $G_{B \pm}$, where one block has eigenvalues that approach 0 as $\rho \rightarrow 0$, while the other block has eigenvalues with real parts bounded away from zero for $\rho$ small.

Proposition 4.4. (a) For $|\zeta|$ small there exists a $C^{\infty}$ matrix $T_{-}(\zeta)$ with a $C^{\infty}$ uniformly bounded inverse such that

$$
T_{-}^{-1} G_{-} T_{-}=\left(\begin{array}{cc}
H_{-}(\zeta)+O\left(\rho^{2}\right) & 0 \\
0 & P_{-}(\zeta)+O(\rho)
\end{array}\right):=G_{B-},
$$

where $H_{-}$is the $n \times n$ matrix given by (3.23) and $P_{-}$is the $(r+2 s) \times(r+2 s)$ matrix

$$
P_{-}=\left(\begin{array}{ccc}
0 & 0 & 1 \\
G_{43} & G_{44} & 0 \\
G_{53} & 0 & G_{55}
\end{array}\right)
$$

with $G_{j k}$ as in (3.9). $T_{-}$is an $(n+r+2 s) \times(n+r+2 s)$ matrix of the form

$$
T_{-}=\left(\begin{array}{ccccc}
1 & 0 & T_{13} & T_{14} & T_{15} \\
0 & 1 & T_{23} & T_{24} & T_{25} \\
0 & 0 & 1 & 0 & 0 \\
H_{21}+\beta_{21} & H_{22}+\beta_{22} & 0 & 1 & 0 \\
0 & 0 & 0 & 0 & 1
\end{array}\right),
$$

where $T_{j k}=O(1), \beta_{2 j}=O\left(\rho^{2}\right)$, and $H_{2 j}$ is an entry of $H_{-}$.

(b) For $|\zeta|$ small there exists a $C^{\infty}$ matrix $T_{+}(\zeta)$ with a $C^{\infty}$ uniformly bounded inverse such that

$$
T_{+}^{-1} G_{+} T_{+}=\left(\begin{array}{cc}
\mathcal{H}_{+}(\zeta)+O\left(\rho^{2}\right) & 0 \\
0 & \mathcal{P}_{+}(\zeta)+O(\rho)
\end{array}\right):=G_{B+}
$$


where $\mathcal{H}_{+}$is the $(n+s) \times(n+s)$ matrix given by

$$
\mathcal{H}_{+}=-\left(A^{d}\left(w_{+}\right)\right)^{-1}\left(\lambda A^{0}+\sum_{j=1}^{d-1} i \eta_{j} A^{j}\right)
$$

and $\mathcal{P}_{+}$is the $(r+s) \times(r+s)$ matrix

$$
\mathcal{P}_{+}=\left(\begin{array}{cc}
\mathcal{G}_{44} & 0 \\
\mathcal{G}_{54} & \mathcal{G}_{55}
\end{array}\right)
$$

with $\mathcal{G}_{j k}$ as in (4.36). $T_{+}$is an $(n+r+2 s) \times(n+r+2 s)$ matrix of the form

$$
T_{+}=\left(\begin{array}{ccccc}
1 & 0 & 0 & \mathcal{T}_{14} & \mathcal{T}_{15} \\
0 & 1 & 0 & \mathcal{T}_{24} & \mathcal{T}_{25} \\
0 & 0 & 1 & \mathcal{T}_{34} & \mathcal{T}_{35} \\
\mathcal{H}_{21}+\alpha_{21} & \mathcal{H}_{22}+\alpha_{22} & \mathcal{H}_{23}+\alpha_{23} & 1 & 0 \\
\mathcal{H}_{31}+\alpha_{31} & \mathcal{H}_{32}+\alpha_{32} & \mathcal{H}_{33}+\alpha_{33} & 0 & 1
\end{array}\right),
$$

where $\mathcal{T}_{j k}=O(1), \alpha_{j k}=O\left(\rho^{2}\right)$, and $\mathcal{H}_{j k}$ is an entry of $\mathcal{H}_{+}$.

Proof. 1. We give the proof for part (a); the argument for part (b) is similar. To motivate the form of $H_{-}$and $P_{-}$in $G_{B-}$, one can do a formal analysis to see that eigenvalues of $G_{-}$ near 0 should be close to eigenvalues of $H_{-}$, while eigenvalues that are bounded away from zero for $\rho$ small should be close to eigenvalues of $P_{-}$.

2. We look for the $O\left(\rho^{2}\right)$ (resp. $\left.O(\rho)\right)$ term in the $H_{-}$block (resp. $P_{-}$block) of $G_{B-}$ in the form:

$$
O\left(\rho^{2}\right)=\left(\begin{array}{ll}
\beta_{11} & \beta_{12} \\
\beta_{21} & \beta_{22}
\end{array}\right) ; O(\rho)=\left(\begin{array}{ccc}
0 & 0 & 0 \\
\delta_{43} & \delta_{44} & \delta_{45} \\
0 & 0 & 0
\end{array}\right)
$$

where the $\beta_{j k}, \delta_{j k}$ are unknowns, along with the $T_{j k}$ in (4.42). To prove the Proposition, it suffices to posit $T_{-}$and $G_{B-}$ of the given forms and use

$$
G_{-} T_{-}=T_{-} G_{B-}
$$

to solve for the above unknowns. Equating corresponding entries in (4.48) yields 25 matrix equations, many of which are satisfied automatically. Below we'll refer to the equation obtained by equating the $(j, k)$ entries of the two sides of $(4.48)$ as the " $(j, k)$-equation". Note, for example, that the 10 equations corresponding to entries in the 3rd and 5th rows are already satisfied, as well as the $(2,1),(2,2)$ equations. The $(4,1)$ equation, for example, is

$$
G_{41}+G_{44}\left(H_{21}+\beta_{21}\right)=\left(H_{21}+\beta_{21}\right)\left(H_{11}+\beta_{11}\right)+\left(H_{22}+\beta_{22}\right)\left(H_{21}+\beta_{21}\right) .
$$

3. We have

$$
\begin{aligned}
& G_{44}=G_{44}^{\#}+O(\rho), \text { where } G_{44}^{\#}=\left(b^{d d}\right)^{-1}\left(a_{22}^{d}-a_{21}^{d}\left(a_{11}^{d}\right)^{-1} a_{12}^{d}\right), \\
& P_{-}=P_{-}^{\#}+O(\rho), \text { where } P_{-}^{\#}=\left(\begin{array}{ccc}
0 & 0 & 1 \\
-\left(b^{d d}\right)^{-1} Q_{2} K & G_{44}^{\#} & 0 \\
\left(D^{d d}\right)^{-1} K & 0 & \left(D^{d d}\right)^{-1} g^{d} I_{s}
\end{array}\right) .
\end{aligned}
$$


We'll use the fact that both $G_{44}^{\#}$ and $P_{-}^{\#}$ are invertible; this follows directly from our structural assumptions.

4. Solve for $\beta_{j k}$. The $(4,1)$ equation is simplified by multiplying the $(1,1)$ equation by $\left(b^{d d}\right)^{-1} a_{21}^{d}$ and subtracting the result from the $(4,1)$ equation. The $(4,2)$ equation is simplified similarly. The $O(\rho)$ terms in the simplified $(4,1)$ and $(4,2)$ equations are seen to cancel out, leaving $O\left(\rho^{2}\right)$ terms. Use the $(1,1)$ and $(1,2)$ equations to define $\beta_{11}$ and $\beta_{12}$ in terms of $\beta_{21}$ and $\beta_{22}$, and substitute these expressions into the $(4,1)$ and $(4,2)$ equations. This gives a nonlinear system for $\beta_{21}$ and $\beta_{22}$ that can be solved by the inverse function theorem for $\rho$ small using the invertibility of $G_{44}^{\#}$.

5. Solve for $T_{j k}, \delta_{j k}$. Use the $(4,3),(4,4),(4,5)$ equations to express the $\delta_{j k}$ in terms of the $T_{j k}$, and substitute these expressions into the $(1,3),(1,4),(1,5)$ and $(2,3),(2,4)$, $(2,5)$ equations. This gives a nonlinear system for the $T_{j k}$ that can be solved by the inverse function theorem for $\rho$ small using the invertibility of $P_{-}^{\#}$.

Definition 4.1. For $\hat{\gamma}>0, \rho>0$ let $E_{ \pm}(\hat{\zeta}, \rho)$ be the space of initial data at $x=0$ of decaying solutions of

$$
U^{\prime}=G(\bar{w}(x), \zeta) U
$$

in $\pm x \geq 0$. Let $F_{ \pm}(\hat{\zeta}, \rho)$ be the space of initial data for decaying solutions of

$$
U^{\prime}=G_{B \pm}(\zeta) U
$$

in $\pm x \geq 0$.

Proposition 4.5. The spaces $E_{ \pm}(\hat{\zeta}, \rho)$ are $C^{\infty}$ in $\hat{\gamma}>0, \rho>0$ and extend continuously to $\hat{\gamma} \geq 0, \rho \geq 0$. We have

$$
\operatorname{dim} E_{+}(\hat{\zeta}, \rho)=r+s ; \operatorname{dim} E_{-}(\hat{\zeta}, \rho)=n+s .
$$

Proof. 1. Let $K_{ \pm}(\hat{\zeta}, \rho)$ be the decaying spaces for $U^{\prime}=G_{ \pm}\left(w_{ \pm}, \zeta\right) U$, and note that

$$
E_{ \pm}(\hat{\zeta}, \rho)=Z_{ \pm}(0, \zeta) K_{ \pm}(\hat{\zeta}, \rho)
$$

The spaces $K_{ \pm}$vary smoothly in $\rho>0, \hat{\gamma}>0$ as a standard consequence of Proposition 4.3 (see [Kat]). By Corollary 4.1 their dimensions are constant on this domain.

Since

$$
E_{ \pm}(\hat{\zeta}, \rho)=Z_{ \pm}(0, \zeta) T_{ \pm}(\zeta) F_{ \pm}(\hat{\zeta}, \rho)
$$

it is enough to prove the remaining statements for $F_{ \pm}(\hat{\zeta}, \rho)$.

2. Dimensions. Using the block diagonal structure of $G_{B \pm}$, we may write with obvious notation

$$
\begin{aligned}
& (a) F_{+}(\hat{\zeta}, \rho)=F_{\mathcal{H}_{+}} \oplus F_{\mathcal{P}_{+}} \\
& (b) F_{-}(\hat{\zeta}, \rho)=F_{H_{-}} \oplus F_{P_{-}} .
\end{aligned}
$$

Since

$$
\mathcal{H}_{+}(\zeta)=\left(\begin{array}{cc}
H_{+}(\zeta) & 0 \\
\mathcal{H}_{21} & -\left(g^{d}\right)^{-1} g^{0} \lambda I_{s}
\end{array}\right)
$$

we deduce from (4.1) that $\operatorname{dim} F_{\mathcal{H}_{+}}=0, \operatorname{dim} F_{H_{-}}=n-1$. 
Recall $G_{44}^{\#}$ from (4.50) and similarly write

$$
\mathcal{G}_{44}=\mathcal{G}_{44}^{\#}+O(\rho)
$$

on the plus side. These matrices arise in the study of viscous profiles for the nonreactive Navier-Stokes equations (see [SZ], e.g.). In Lemma 2 of [SZ], it is shown that our hypotheses (H0) and (H7) imply that the number of eigenvalues of $G_{44}^{\#}$ with $\Re \mu<0$ is equal to $r+p-q$, where $p$ is the number of positive eigenvalues of $\left(a_{11}^{0}\right)^{-1} a_{11}^{d}$ (zero by (H5)), and $q$ is the number of positive eigenvalues of $\left(a^{0}\right)^{-1} a^{d}\left(V_{-}\right)$(one by $(\mathrm{H} 0)$ ). Thus, $G_{44}^{\#}$ (resp. $\mathcal{G}_{44}^{\#}$ ) has one (resp., no) eigenvalue with positive real part. From (4.45) and (H1),(H3) we deduce immediately that

$$
\operatorname{dim} F_{\mathcal{P}_{+}}=r+s
$$

Knowing $G_{44}^{\#}$ has one eigenvalue with $\Re \mu>0$, we obtain

$$
\operatorname{dim} F_{P_{-}}=s+1
$$

as follows. A simple computation shows that $\mu$ is an eigenvalue of $P_{-}^{\#}$ if and only if either

$$
\begin{aligned}
& \text { (a) } \operatorname{det}\left(\mu^{2} D^{d d}-\mu g^{d} I_{s}-K\right)=0 \text { or } \\
& \text { (b) } \mu \text { is an eigenvalue of } G_{44}^{\#} \text {. }
\end{aligned}
$$

Using (H7), replacing $K$ by $K+m I_{s}$ and taking $m>0$ large, we deduce there are $s$ roots of (4.61)(a) with $\Re \mu>0$ and $s$ roots with $\Re \mu<0$.

3. Continuous extension. Since both $\mathcal{P}_{+}(\zeta)$ and $P_{-}(\zeta)$ have no center subspace for $\rho$ small, $F_{\mathcal{P}_{+}}$and $F_{P_{-}}$clearly extend smoothly to $\hat{\gamma} \geq 0, \rho \geq 0$. Also, $F_{\mathcal{H}_{+}} \equiv 0$ is a smooth extension of $F_{\mathcal{H}_{+}}$.

The continuous extension of $F_{H_{-}}$is quite nontrivial, mainly because of the presence of glancing modes. The existence of such an extension is the main result, Theorem 1.1, of [MZ2]. The proof requires a further (microlocal) conjugation of the $H_{-}+O\left(\rho^{2}\right)$ block of $G_{B-}$ to generalized block structure in the sense of Lemma 2.10 of [MZ1], and the construction of Kreiss-type symmetrizers. We remark that our constant multiplicity assumption (H6) is used in the conjugation to generalized block structure.

Fixing a basepoint $\hat{\zeta}^{*} \in S_{+}^{d}$, we now construct bases for the spaces of decaying solutions of (4.34) in $\pm x \geq 0$ for $\rho>0, \hat{\zeta}>0$ and $(\hat{\zeta}, \rho)$ near $\left(\hat{\zeta}^{*}, 0\right)$. Let us rewrite the upper left block of $G_{B-}$

$$
H_{-}(\zeta)+O\left(\rho^{2}\right):=\rho \hat{H}_{-}(\hat{\zeta}, \rho), \text { where } \hat{H}_{-}(\hat{\zeta}, 0)=H_{-}(\hat{\zeta})
$$

The continuous extendibility of $F_{H_{-}}(\hat{\zeta}, \rho)$ implies that we can choose a local basis for $F_{H_{-}}$

$$
\mathrm{s}_{-}^{j}(\hat{\zeta}, \rho), j=1, \ldots, n-1
$$

that extends continuously to $\hat{\gamma} \geq 0, \rho \geq 0$ and satisfies

$$
\mathrm{s}_{-}^{j}(\hat{\zeta}, 0)=s_{-}^{j}(\hat{\zeta})
$$


for $s_{-}^{j}(\hat{\zeta})$ as in (4.2). In $x \leq 0$ there are $n-1$ slow modes

$$
U_{-}^{j}(x, \hat{\zeta}, \rho)=Z_{-}(x, \zeta) T_{-}(\zeta)\left(\begin{array}{c}
e^{x \rho \hat{H}_{-}(\hat{\zeta}, \rho)} \mathbf{s}_{-}^{j}(\hat{\zeta}, \rho) \\
0
\end{array}\right), j=1, \ldots, n-1
$$

and $s+1$ fast modes

$$
U_{-}^{j}(x, \zeta)=Z_{-}(x, \zeta) T_{-}(\zeta)\left(\begin{array}{c}
0 \\
e^{x P_{-}(\zeta)} \pi(\zeta) c^{j}
\end{array}\right), j=n, \ldots, n+s,
$$

where the $c^{j}$ give a basis of $F_{P_{-}(0)}$ and $\pi(\zeta)$ denotes projection onto the positive generalized eigenspace of $P_{-}(\zeta)$. Similarly, in $x \geq 0$ there are $r+s$ fast modes

$$
U_{+}^{j}(x, \zeta)=Z_{+}(x, \zeta) T_{+}(\zeta)\left(\begin{array}{c}
0 \\
e^{x \mathcal{P}_{+}(\zeta)} \tilde{\pi}(\zeta) d^{j}
\end{array}\right), j=1, \ldots, r+s,
$$

where the $d^{j}$ give a basis of $F_{\mathcal{P}_{+}(0)}$ and $\tilde{\pi}(\zeta)$ denotes projection onto the negative generalized eigenspace of $\mathcal{P}_{+}(\zeta)$.

Remark 4.6. 1. The construction shows that fast modes are $C^{\infty}$ in $\zeta$ for $|\zeta|$ small, while slow modes are $C^{\infty}$ in $(\hat{\zeta}, \rho)$ for $\hat{\gamma}>0, \rho>0$, and $\hat{\zeta}$ near $\hat{\zeta}^{*}$, with continuous extensions to $\hat{\gamma} \geq 0$, $\rho \geq 0$.

2. Although we are not interested in the behavior of $U_{ \pm}^{j}$ in $\mp x \geq 0$, it is convenient to extend the modes to all of $\mathbb{R}_{x}$ as solutions of (4.34); henceforth, we assume this has been done. Note that the formulas (4.65)-(4.67) no longer hold on the extended domain.

We may now define the RNS Evans function by observing that the linearized problem (4.34) has solutions decaying as $x \rightarrow \pm \infty$ and growing exponentially in time (for $(\hat{\zeta}, \rho)$ near $\left.\left(\hat{\zeta}^{*}, 0\right)\right)$ if and only if for some $\gamma>0$ the following $(n+r+2 s) \times(n+r+2 s)$ determinant vanishes:

$$
D_{R N S}(\hat{\zeta}, \rho)=\left.\operatorname{det}\left(U_{-}^{1}, \ldots, U_{-}^{n+s}, U_{+}^{1}, \ldots, U_{+}^{r+s}\right)\right|_{x=0}
$$

Remark 4.7. 1. Using wedge products as in Remarks 4.1 and 4.2, we obtain a function $D_{R N S}(\hat{\zeta}, \rho)$ defined on $S_{+}^{d} \times\left[0, \rho_{0}\right]$ for $\rho_{0}$ small that is $C^{\infty}$ in $(\hat{\zeta}, \rho)$ for $\hat{\gamma}>0, \rho>0$, with a continuous extension to $\hat{\gamma} \geq 0, \rho \geq 0$. The analysis in section 6 will show that the same is true for $D_{R N S} / \rho$.

2. For $\hat{\gamma} \geq 0, \rho>0$ Proposition 4.3 shows that the matrices $G_{ \pm}\left(w_{ \pm}, \zeta\right)$ have no pure imaginary eigenvalues. Thus, we can define $D_{R N S}$ locally near any basepoint $P^{*}$ in $\hat{\gamma} \geq 0$, $\rho>0$ by the above formula (4.68), except that we now replace the modes $U_{ \pm}^{j}$ used there by

$$
\begin{aligned}
& U_{-}^{j}(x, \zeta)=Z_{-}(x, \zeta) \mathcal{U}_{-}^{j}(x, \zeta), j=1, \ldots, n+s, \\
& U_{+}^{j}(x, \zeta)=Z_{+}(x, \zeta) \mathcal{U}_{+}^{j}(x, \zeta), j=1, \ldots, r+s,
\end{aligned}
$$

where the $\mathcal{U}_{ \pm}^{j}$ are (fast) decaying solutions to the limiting problems in $\pm x \geq 0$ defined for $\zeta$ near $P^{*}$. Using wedge products and analytic continuation as before, $D_{R N S}$ can be extended real analytically to all of $\hat{\gamma} \geq 0, \rho>0$ with a continuous extension to $\hat{\gamma} \geq 0, \rho \geq 0$.

Proposition 4.6. Slow modes satisfy

$$
\lim _{x \rightarrow-\infty} U_{-}^{j}(x, \hat{\zeta}, 0)=\left(\begin{array}{c}
s_{-}^{j}(\hat{\zeta}) \\
0
\end{array}\right)
$$


while fast modes in $x \leq 0$ satisfy

$$
\lim _{x \rightarrow-\infty} U_{-}^{j}(x, 0)=\left(\begin{array}{l}
0 \\
0
\end{array}\right) \quad \text { (exponentially fast); }
$$

fast modes in $x \geq 0$ decay similarly.

Proof. The proof follows directly from the formulas for slow and fast modes, using the properties of $Z_{ \pm}$and the explicit structure of $T_{ \pm}$.

For the analysis in the next section we make a special choice of the fast modes $U_{-}^{n+s}$ and $U_{+}^{r+s}$. Differentiating the RNS profile equation (1.5) shows that $\bar{w}^{\prime}(x)=\left(\bar{v}_{1}^{\prime}, \bar{v}_{2}^{\prime}, \overline{\mathrm{y}}^{\prime}\right)$ satisfies the linearized, transformed problem (3.3) when $\zeta=0$. Thus,

$$
\bar{U}(x)=\left(\bar{V}_{1}^{\prime}, \bar{V}_{2}^{\prime}, \bar{Y}^{\prime}, \bar{V}_{2}^{\prime \prime}, \bar{Y}^{\prime \prime}\right)
$$

is a solution of $U^{\prime}=G(\bar{w}(x), 0) U$ on $\mathbb{R}_{x}$ that is exponentially decaying as $x \rightarrow \pm \infty$.

The next Proposition is proved just like Proposition 4.2.

Proposition 4.7. There exist $\bar{c} \in \mathbb{C}^{r+2 s}, \bar{d} \in \mathbb{C}^{r+s}$ such that the fast modes given by

$$
\begin{aligned}
& U_{-}^{n+s}(x, \zeta)=Z_{-}(x, \zeta) T_{-}(\zeta)\left(\begin{array}{c}
0 \\
e^{x P_{-}(\zeta)} \pi(\zeta) \bar{c}
\end{array}\right) \text { in } x \leq 0 \\
& U_{+}^{r+s}(x, \zeta)=Z_{+}(x, \zeta) T_{+}(\zeta)\left(\begin{array}{c}
0 \\
e^{x \mathcal{P}_{+}(\zeta)} \tilde{\pi}(\zeta) \bar{d}
\end{array}\right) \text { in } x \geq 0
\end{aligned}
$$

are $C^{\infty}$ and satisfy

$$
U_{-}^{n+s}(x, 0)=\bar{U}(x) \text { in } x \leq 0 ; \quad U_{+}^{r+s}(x, 0)=\bar{U}(x) \text { in } x \geq 0 .
$$

\section{Part 4. The Equivalence Theorem}

In this part we compute the low frequency expansions of $D_{Z N D}(\hat{\zeta}, \rho)$ and $D_{R N D}(\hat{\zeta}, \rho)$ and prove the Equivalence Theorem.

\section{LOW FREQUENCY EXPANSION FOR ZND}

Recall the linearized, transformed interior equation in $x<0$ (3.18):

$$
\text { (a) }\left(\lambda A^{0}(\tilde{w})+\sum_{j=1}^{d-1} i \eta_{j} A^{j}(\tilde{w})\right) w+\left(A^{d}(\tilde{w}) w\right)^{\prime}=d_{w} R(\tilde{w}) w,
$$

where we've dropped the \# on $w=(v, \mathrm{y})$. By examining (5.1) we will find a row operation that can be used to simplify the ZND determinant.

Writing out the two components of equation (5.1) and then combining them by taking $Q($ second $)+$ first, we obtain

$$
\begin{gathered}
\left(a^{d}(\tilde{V}) v+Q d_{V} g^{d}(\tilde{V}) v \tilde{Y}+Q g^{d}(\tilde{V}) \mathrm{y}\right)^{\prime}+\left(\lambda a^{0}(\tilde{V})+\sum_{j=1}^{d-1} i \eta_{j} a^{j}(\tilde{V})\right) v+ \\
Q \lambda\left(d_{V} g^{0}(\tilde{V}) v \tilde{Y}+g^{0}(\tilde{V}) \mathrm{y}\right)+Q \sum_{j=1}^{d-1} i \eta_{j} d_{V} g^{j}(\tilde{V}) v \tilde{Y}=0 .
\end{gathered}
$$


At $\rho=0$ (5.2) shows that the quantity

$$
\mathcal{R}(v, \mathrm{y}):=a^{d}(\tilde{V}) v+Q d_{V} g^{d}(\tilde{V}) v \tilde{Y}+Q g^{d}(\tilde{V}) \mathrm{y}
$$

is independent of $x$ in $x<0$. When $w$ is taken to be one of the slow or fast modes $w^{j}$, we can easily compute $\mathcal{R} w^{j}(x, \hat{\zeta}, 0)$ :

Lemma 5.1. We have for $x \leq 0$ :

$$
\begin{aligned}
& \mathcal{R} w^{j}(x, \hat{\zeta}, 0)=a^{d}\left(V_{-}\right) s_{-}^{j}=r_{-}^{j}(\hat{\zeta}) \text { for slow modes } j=1, \ldots, n-1 \\
& \mathcal{R} w^{j}(x, 0)=0 \text { for fast modes } j=n, \ldots, n-1+s
\end{aligned}
$$

Proof. Integrate (5.2) $\int_{-\infty}^{x}$ at $\rho=0$, and use Proposition 4.1 together with the fact that $\tilde{Y}(-\infty)=Y_{-}=0$.

For later use, we note that the regularity of slow modes evident in the explicit expression (4.18) implies

$$
\mathcal{R} w^{j}(x, \hat{\zeta}, \rho)=r_{-}^{j}(\hat{\zeta})+O(\rho), j=1, \ldots, n-1 .
$$

The jump term in the CJ determinant is a jump between the states $w_{+}$and $w_{-}$, while the jump term in $D_{Z N D}$ involves the states $w_{+}$and $w_{*}$. We can understand the relation between these jumps by considering the variation of $w^{n-1+s}(4.33)$ at $\rho=0$. Let

$$
\mathbb{W}(x, \hat{\zeta}, 0)=\left(\begin{array}{l}
\mathbb{V} \\
\mathbb{Y}
\end{array}\right)=\left.\partial_{\rho}\right|_{\rho=0} w^{n-1+s}(x, \hat{\zeta}, \rho) .
$$

For any function $h(\tilde{w})$ let

$$
[h(\tilde{w})]_{!}=h\left(w_{*}\right)-h\left(w_{-}\right) \text {and recall }[h(\tilde{w})]=h\left(w_{+}\right)-h\left(w_{-}\right) .
$$

Proposition 5.1. We have at $x=0^{-}, \rho=0$

$$
\mathcal{R} \mathbb{W}\left(0^{-}, \hat{\zeta}, 0\right)=-\left(\hat{\lambda}\left[f^{0}(\tilde{V})\right]_{!}+i\left[f^{\hat{\eta}}(\tilde{V})\right] !+\hat{\lambda} Q\left[g^{0}(\tilde{V}) \tilde{Y}\right] !\right)
$$

Proof. Take $(v, \mathrm{y})=\left(v^{n-1+s}, \mathrm{y}^{n-1+s}\right)$ in $(5.2)$, write $\zeta=\rho(\hat{\lambda}, \hat{\eta})$, and apply $\left.\partial_{\rho}\right|_{\rho=0}$ to equation (5.2) to obtain

$$
\begin{gathered}
\left(a^{d}(\tilde{V}) \mathbb{V}+Q d_{V} g^{d}(\tilde{V}) \mathbb{V} \tilde{Y}+Q g^{d}(\tilde{V}) \mathbb{Y}\right)^{\prime}+\left(\hat{\lambda} a^{0}(\tilde{V})+\sum_{j=1}^{d-1} i \hat{\eta}_{j} a^{j}(\tilde{V})\right) \tilde{V}^{\prime}+ \\
Q \hat{\lambda}\left(d_{V} g^{0}(\tilde{V}) \tilde{V}^{\prime} \tilde{Y}+g^{0}(\tilde{V}) \tilde{Y}^{\prime}\right)=0
\end{gathered}
$$

where we've used

$$
d_{V} g^{j}(\tilde{V}) \tilde{V}^{\prime}=\left(g^{j}(\tilde{V})\right)^{\prime}=0, j=1, \ldots, d-1 .
$$

The left side of (5.9) is a perfect derivative; integrating $\int_{-\infty}^{0}$ immediately gives the result.

Corollary 5.1. We have

$$
\mathcal{R} w^{n-1+s}\left(0^{-}, \hat{\zeta}, \rho\right)=-\rho\left(\hat{\lambda}\left[f^{0}(\tilde{V})\right] !+i\left[f^{\hat{\eta}}(\tilde{V})\right] !+\hat{\lambda} Q\left[g^{0}(\tilde{V}) \tilde{Y}\right] !\right)+O\left(\rho^{2}\right)
$$


Proof. Apply $\mathcal{R}$ to the equation

$$
w^{n-1+s}(x, \hat{\zeta}, \rho)=\tilde{w}^{\prime}(x)+\rho \mathbb{W}(x, \hat{\zeta}, 0)+O\left(\rho^{2}\right),
$$

using Proposition 5.1 and the fact that $\mathcal{R} \tilde{w}^{\prime}(x)=0$ (3.19).

Next we collect a few pieces of notation that will be used in the analysis of $D_{Z N D}$.

\section{Notation 5.1.}

1. $\alpha=\hat{\lambda}\left[f^{0}(\tilde{V})\right]_{*}+i\left[f^{\hat{\eta}}(\tilde{V})\right]_{*}$

2. $\beta=\hat{\lambda}\left[g^{0}(\tilde{V}) \tilde{Y}\right]_{*}+\mathcal{Z}(\hat{\zeta}) ;$ recall $(4.23)$

3. $W_{1}=\hat{\lambda}\left[f^{0}(\tilde{V})\right]+i\left[f^{\hat{\eta}}(\tilde{V})\right]+\hat{\lambda} Q\left[g^{0}(\tilde{V}) \tilde{Y}\right]$

4. $W_{2}=\hat{\lambda}\left[f^{0}(\tilde{V})\right]_{*}+i\left[f^{\hat{\eta}}(\tilde{V})\right]_{*}+\hat{\lambda} Q\left[g^{0}(\tilde{V}) \tilde{Y}\right]_{*}=\alpha+\hat{\lambda} Q\left[g^{0}(\tilde{V}) \tilde{Y}\right]_{*}$

5. For $v \in \mathbb{C}^{n}$ let $N v=Q d_{V} g^{d}\left(V_{*}\right) v Y_{*} \in \mathbb{C}^{n}$.

6. Set $a^{d}\left(V_{*}\right)=a^{d}, g^{d}\left(V_{*}\right)=g^{d}, r_{-}^{j}(\hat{\zeta})=r^{j}, \kappa=\left(\operatorname{det} a^{d}\right)\left(\operatorname{det}\left(a^{d}+N\right)\right)^{-1}$.

Observe that we have

$$
\begin{aligned}
& \text { (a) } W_{1}-W_{2}=\hat{\lambda}\left[f^{0}(\tilde{V})\right]_{!}+i\left[f^{\hat{\eta}}(\tilde{V})\right]_{!}+\hat{\lambda} Q\left[g^{0}(\tilde{V}) \tilde{Y}\right]_{!}=-\mathcal{R} \mathbb{W}\left(0^{-}, \hat{\zeta}, 0\right) \\
& \text { (b) }\left(I+N a^{d}\left(V_{*}\right)^{-1}\right) \alpha+Q \beta=W_{2} .
\end{aligned}
$$

Theorem 5.1. Under hypotheses (HO), (H1), (H2), (H4), (H6), and (H8), the ZND Evans function satisfies

$$
\begin{aligned}
D_{Z N D}(\hat{\zeta}, \rho)= & \rho \beta_{1} \operatorname{det}\left(r_{-}^{1}(\hat{\zeta}), \ldots, r_{-}^{n-1}(\hat{\zeta}), \hat{\lambda}\left[f^{0}(\tilde{V})\right]+i\left[f^{\hat{\eta}}(\tilde{V})\right]+\hat{\lambda} Q\left[g^{0}(\tilde{V}) \tilde{Y}\right]\right)+O\left(\rho^{2}\right)= \\
& \rho \beta_{1} \Delta_{C J}(\hat{\zeta}, 1)+O\left(\rho^{2}\right),
\end{aligned}
$$

where

$$
\beta_{1}=\left.(-1)^{s}\left(g^{d}\left(V_{*}\right)\right)^{s} \kappa \operatorname{det}\left(y^{n}, \ldots, y^{n-1+s}\right)\right|_{x=0^{-}, \zeta=0},
$$

and the error is $O\left(\rho^{2}\right)$ uniformly for $\hat{\zeta} \in S_{+}^{d}=\{\hat{\zeta}:|\hat{\zeta}|=1, \hat{\gamma} \geq 0\}$.

Proof. Starting from (4.25) we compute:

$$
\begin{aligned}
& D_{Z N D}(\hat{\zeta}, \rho)=\left.\operatorname{det}\left(\begin{array}{llll}
a^{d} v^{1} & \cdots & a^{d} v^{n-1+s} & \rho \alpha+a^{d} \tilde{V}^{\prime} \\
g^{d} \mathbf{y}^{1} & \cdots & g^{d} \mathbf{y}^{n-1+s} & \rho \beta+g^{d} \tilde{Y}^{\prime}
\end{array}\right)\right|_{x=0^{-}}= \\
& \left.\operatorname{det}\left(\begin{array}{llll}
a^{d} v^{1} & \ldots & a^{d} v^{n-1+s} & \rho \alpha-\rho a^{d} \mathbb{V}+O\left(\rho^{2}\right) \\
g^{d} \mathbf{y}^{1} & \cdots & g^{d} \mathbf{y}^{n-1+s} & \rho \beta-\rho g^{d} \mathbb{Y}+O\left(\rho^{2}\right)
\end{array}\right)\right|_{x=0^{-}}= \\
& \left.\rho \operatorname{det}\left(\begin{array}{llll}
a^{d} v^{1} & \cdots & a^{d} v^{n-1+s} & \alpha-a^{d} \mathbb{V}+O(\rho) \\
g^{d} \mathbf{y}^{1} & \cdots & g^{d} \mathbf{y}^{n-1+s} & \beta-g^{d} \mathbb{Y}+O(\rho)
\end{array}\right)\right|_{x=0^{-}}= \\
& \left.\rho \operatorname{det} a^{d} \operatorname{det}\left(\begin{array}{cccc}
v^{1} & \cdots & v^{n-1+s} & \left(a^{d}\right)^{-1}\left(\alpha-a^{d} \mathbb{V}+O(\rho)\right) \\
g^{d} \mathbf{y}^{1} & \cdots & g^{d} \mathbf{y}^{n-1+s} & \beta-g^{d} \mathbb{Y}+O(\rho)
\end{array}\right)\right|_{x=0^{-}}
\end{aligned}
$$

where to obtain the second equality we have subtracted the $(n-1+s)$-th column from the last and used (5.12).

Next apply the row operation

$$
\mathcal{R}\left(\begin{array}{l}
v \\
\mathrm{y}
\end{array}\right)\left(0^{-}, \hat{\zeta}, \rho\right)=\left(a^{d}+N\right) v+Q g^{d} \mathbf{y}
$$


and use Lemma 5.1, (5.5), and $(5.13)(\mathrm{b})$ to obtain at $x=0^{-}$;

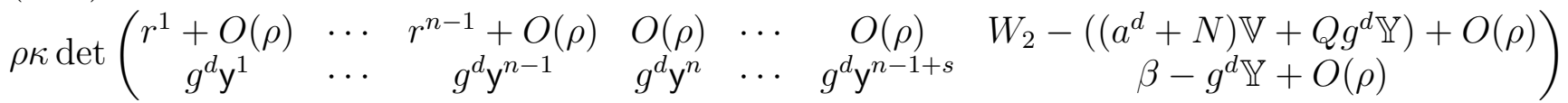

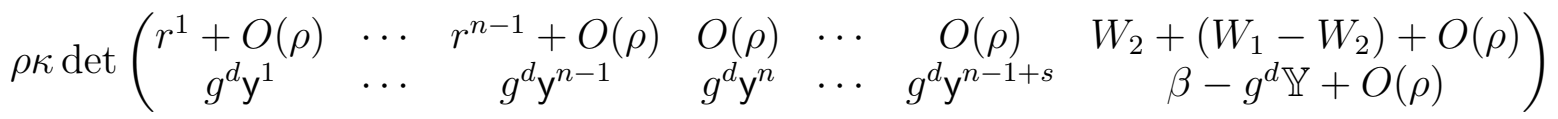

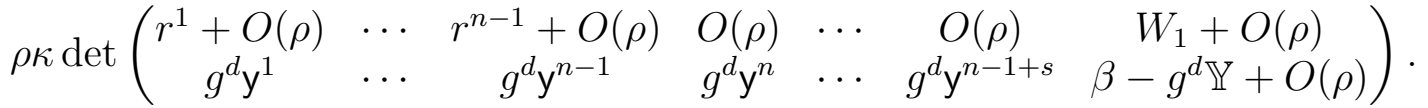

Expanding the determinant about $\rho=0$, we obtain (5.14).

The uniformity of the error $O\left(\rho^{2}\right)$ with respect to $\hat{\zeta} \in S_{+}^{d}$ is evident from the regularity that can be read off from the explicit formulas for slow and fast modes (4.18), (4.19).

Remark 5.1. The nonvanishing of the transversality constant $\beta_{1}$ for the physical ZND equations is discussed in section 8 of part 5 .

\section{LOW FREQUENCY EXPANSION FOR RNS}

In this section we complete the proof of the Equivalence Theorem, Theorem 0.1. First, for easy reference we gather here some of the notation used in this section.

Notation 6.1. Unless otherwise indicated, in this section all matrix coefficients are evaluated at the profile $\bar{w}=(\bar{V}, \bar{Y})$.

1. Let

$$
\begin{aligned}
\mathcal{B}^{j k} & =\left(\begin{array}{cc}
0 & 0 \\
0 & b^{j k}
\end{array}\right), j, k=1, \ldots, d \\
\mathcal{A}^{j} v & =a^{j} v-\left(v \cdot d_{V} \mathcal{B}^{j d}\right) \bar{V}^{\prime}, j=0, \ldots, d \text { where } \mathcal{B}^{0 d}=0 .
\end{aligned}
$$

2. Let

$$
P^{j} \mathrm{y}+E^{j} v:=d_{V} g^{j} \bar{Y} v+g^{j} \mathrm{y}-\left(v \cdot d_{V} D^{j d}\right) \bar{Y}^{\prime}, j=0, \ldots, d,
$$

where $D^{0 d}=0$ and $g^{j}(\bar{V})=0$ for $j=1, \ldots, d-1$.

3. Define the $(n+s) \times(n+s)$ matrix $\mathcal{N}$ and the $n \times n$ matrix $C$

$$
\mathcal{N}=\left(\begin{array}{ccc}
a_{11}^{d} & a_{12}^{d} & 0 \\
0 & b^{d d} & 0 \\
0 & 0 & D^{d d}
\end{array}\right):=\left(\begin{array}{cc}
C & 0 \\
0 & D^{d d}
\end{array}\right) .
$$

4. Let

$$
\begin{aligned}
& \mathcal{K}=\left(D^{d d}\right)^{-1} \\
& H_{*}=H\left(C^{-1}\right) \text { for any } n \times n \text { matrix } H .
\end{aligned}
$$

5. Define the change of variables

$$
z=\left(\begin{array}{c}
z_{1} \\
z_{2} \\
z_{3}
\end{array}\right):=\mathcal{N}\left(\begin{array}{c}
v_{1} \\
v_{2} \\
\mathrm{y}
\end{array}\right) ; \quad Z:=\left(z_{1}, z_{2}, z_{3}, z_{2}^{\prime}, z_{3}^{\prime}\right)^{t} .
$$

and set $z_{\mathrm{b}}=\left(z_{1}, z_{2}\right)$, so $v=C^{-1} z_{\mathrm{b}}$ and $\mathrm{y}=\mathcal{K} z_{3}$. 
The Evans function $D_{R N S}$ can be expressed in terms of the $z$ coordinates as follows. Let

$$
z_{ \pm}^{j}=\mathcal{N}\left(\begin{array}{c}
v_{ \pm}^{j} \\
y_{ \pm}^{j}
\end{array}\right)
$$

for fast or slow modes $\left(\begin{array}{c}v_{ \pm}^{j} \\ y_{ \pm}^{j}\end{array}\right)$ as defined in (4.65)-(4.67), and let $Z_{ \pm}^{j}$ be the corresponding enlarged vectors as in (6.5). A simple computation shows

$$
\begin{aligned}
& \tilde{D}_{R N S}:=\left.\operatorname{det}\left(Z_{-}^{1}, \ldots, Z_{-}^{n+s}, Z_{+}^{1}, \ldots, Z_{+}^{r+s}\right)\right|_{x=0}= \\
& \left.\quad \kappa \operatorname{det}\left(U_{-}^{1}, \ldots, U_{-}^{n+s}, U_{+}^{1}, \ldots, U_{+}^{r+s}\right)\right|_{x=0}=\kappa D_{R N S},
\end{aligned}
$$

where $\kappa=\left.\left(\operatorname{det} A^{d} \cdot \operatorname{det} b^{d d} \cdot \operatorname{det} D^{d d}\right)\right|_{x=0} \neq 0$.

We proceed to examine the linearized, transformed RNS equations (3.3) in order to find row operations to use in simplifying the determinant $\tilde{D}_{R N S}$. Writing frequencies in polar coordinates, we obtain for the components of (3.3):

$$
\begin{aligned}
& \left(\mathcal{B}^{d d} v^{\prime}\right)^{\prime}=\rho \hat{\lambda} \mathcal{A}^{0} v+\rho \sum_{j=1}^{d-1} i \hat{\eta}_{j} \mathcal{A}^{j} v+\left(\mathcal{A}^{d} v\right)^{\prime} \\
& -\rho \sum_{j=1}^{d-1} i \hat{\eta}_{j} \mathcal{B}^{j d} v^{\prime}-\rho \sum_{k=1}^{d-1} i \hat{\eta}_{k}\left(\mathcal{B}^{d k} v\right)^{\prime}+\rho^{2} \sum_{j, k=1}^{d-1} \mathcal{B}^{j k} \hat{\eta}_{j} \hat{\eta}_{k} v-Q K\left(d_{V} \psi \bar{Y} v+\psi y\right) \\
& \left(D^{d d} \mathbf{y}^{\prime}\right)^{\prime}=\rho \hat{\lambda}\left(P^{0} \mathbf{y}+E^{0} v\right)+\rho \sum_{j=1}^{d-1} i \hat{\eta}_{j}\left(P^{j} \mathbf{y}+E^{j} v\right)+\left(P^{d} \mathbf{y}+E^{d} v\right)^{\prime} \\
& -\rho \sum_{j=1}^{d-1} i \hat{\eta}_{j} D^{j d} \mathbf{y}^{\prime}-\rho \sum_{k=1}^{d-1} i \hat{\eta}_{k}\left(D^{d k} \mathbf{y}\right)^{\prime}+\rho^{2} \sum_{j, k=1}^{d-1} D^{j k} \hat{\eta}_{j} \hat{\eta}_{k} \mathbf{y}+K\left(d_{V} \psi \bar{Y} v+\psi y\right) .
\end{aligned}
$$

Rewriting in terms of $z$ coordinates gives

(a) $\left(\mathcal{B}_{*}^{d d} z_{b}^{\prime}\right)^{\prime}=\rho \hat{\lambda} \mathcal{A}_{*}^{0} z_{b}+\rho \sum_{j=1}^{d-1} i \hat{\eta}_{j}\left(\mathcal{A}_{*}^{j}-\mathcal{B}^{j d}\left(C^{-1}\right)^{\prime}\right) z_{b}+\left(\left(\mathcal{A}_{*}^{d}-\mathcal{B}^{d d}\left(C^{-1}\right)^{\prime}\right) z_{b}\right)^{\prime}$

$$
-\rho \sum_{j=1}^{d-1} i \hat{\eta}_{j} \mathcal{B}_{*}^{j d} z_{b}^{\prime}-\rho \sum_{k=1}^{d-1} i \hat{\eta}_{k}\left(\mathcal{B}_{*}^{d k} z_{b}\right)^{\prime}+\rho^{2} \sum_{j, k=1}^{d-1} \mathcal{B}_{*}^{j k} \hat{\eta}_{j} \hat{\eta}_{k} z_{b}-Q K\left(d_{V} \psi \bar{Y} C^{-1} z_{b}+\psi \mathcal{K} z_{3}\right)
$$

(b) $\left(D^{d d}\left(\mathcal{K} z_{3}\right)^{\prime}\right)^{\prime}=\rho \hat{\lambda}\left(P^{0} \mathcal{K} z_{3}+E_{*}^{0} z_{b}\right)+\rho \sum_{j=1}^{d-1} i \hat{\eta}_{j}\left(P^{j} \mathcal{K} z_{3}+E_{*}^{j} z_{b}\right)+\left(P^{d} \mathcal{K} z_{3}+E_{*}^{d} z_{b}\right)^{\prime}$

$$
-\rho \sum_{j=1}^{d-1} i \hat{\eta}_{j} D^{j d}\left(\mathcal{K} z_{3}\right)^{\prime}-\rho \sum_{k=1}^{d-1} i \hat{\eta}_{k}\left(D^{d k} \mathcal{K} z_{3}\right)^{\prime}+\rho^{2} \sum_{j, k=1}^{d-1} D^{j k} \hat{\eta}_{j} \hat{\eta}_{k} \mathcal{K} z_{3}+K\left(d_{V} \psi \bar{Y} C^{-1} z_{b}+\psi \mathcal{K} z_{3}\right) .
$$

Setting $\rho=0$ and combining the equations (6.9) in the obvious way gives

$$
\left(\mathcal{B}_{*}^{d d} z_{b}^{\prime}\right)^{\prime}=\left(\left(\mathcal{A}_{*}^{d}-\mathcal{B}^{d d}\left(C^{-1}\right)^{\prime}\right) z_{b}\right)^{\prime}-Q\left\{\left(D^{d d}\left(\mathcal{K} z_{3}\right)^{\prime}\right)^{\prime}-\left(P^{d} \mathcal{K} z_{3}+E_{*}^{d} z_{b}\right)^{\prime}\right\} .
$$


When $z$ is a fast mode on $x \leq 0$ (i.e., $z=\mathcal{N}\left(\begin{array}{l}v_{-}^{j} \\ \mathrm{y}_{-}^{j}\end{array}\right)$ for a mode as in (4.66)), we integrate (6.10) $\int_{-\infty}^{x}$ and use Proposition 4.6 to get

$$
\mathcal{B}_{*}^{d d} z_{b}^{\prime}=\left(\mathcal{A}_{*}^{d}-\mathcal{B}^{d d}\left(C^{-1}\right)^{\prime}\right) z_{b}-Q\left\{D^{d d}\left(\mathcal{K} z_{3}\right)^{\prime}-\left(P^{d} \mathcal{K} z_{3}+E_{*}^{d} z_{b}\right)\right\}
$$

For fast modes on $x \geq 0$ we integrate $\int_{+\infty}^{x}$ to get an equation that looks the same. For slow modes integration on $\int_{-\infty}^{x}$ yields

$$
\mathcal{B}_{*}^{d d} z_{b}^{\prime}=\left(\mathcal{A}_{*}^{d}-\mathcal{B}^{d d}\left(C^{-1}\right)^{\prime}\right) z_{b}-Q\left\{D^{d d}\left(\mathcal{K} z_{3}\right)^{\prime}-\left(P^{d} \mathcal{K} z_{3}+E_{*}^{d} z_{b}\right)\right\}-r_{-}^{j}(\hat{\zeta}),
$$

for $r_{-}^{j}$ as in (4.4).

Using the explicit forms of the matrices defined in Notation 6.1, we easily compute

$$
\mathcal{B}_{*}^{d d}=\left(\begin{array}{cc}
0 & 0 \\
0 & I_{r}
\end{array}\right) ; \mathcal{A}_{*}^{d}-\mathcal{B}^{d d}\left(C^{-1}\right)^{\prime}=\left(\begin{array}{cc}
I_{n-r} & 0 \\
M_{1} & M_{2}
\end{array}\right),
$$

for some $M_{1}, M_{2}$. Taking components in (6.11) (recall $Q=\left(\begin{array}{c}0 \\ Q_{2}\end{array}\right)$ ) gives

$$
\begin{aligned}
& 0=z_{1} \\
& z_{2}^{\prime}=M_{1} z_{1}+M_{2} z_{2}-\left\{Q\left(D^{d d}\left(\mathcal{K} z_{3}\right)^{\prime}-\left(P^{d} \mathcal{K} z_{3}+E_{*}^{d} z_{b}\right)\right)\right\}_{2},
\end{aligned}
$$

and doing the same in (6.12) gives

$$
\begin{aligned}
& 0=z_{1}-\left(r_{-}^{j}(\hat{\zeta})\right)_{1} \\
& z_{2}^{\prime}=M_{1} z_{1}+M_{2} z_{2}-\left\{Q\left(D^{d d}\left(\mathcal{K} z_{3}\right)^{\prime}-\left(P^{d} \mathcal{K} z_{3}+E_{*}^{d} z_{b}\right)\right)\right\}_{2}-\left(r_{-}^{j}(\hat{\zeta})\right)_{2} .
\end{aligned}
$$

The final step before computing $\tilde{D}_{R N S}$ is to derive the analogue of $(6.14),(6.15)$ for the jump in the variation of the profile

$$
\begin{aligned}
& \mathbb{Z}(x, \hat{\zeta}):=\mathbb{Z}_{-}-\mathbb{Z}_{+}, \text {where } \\
& \mathbb{Z}_{-}(x, \hat{\zeta})=\left.\partial_{\rho}\right|_{\rho=0} Z_{-}^{n+s}, \quad \mathbb{Z}_{+}(x, \hat{\zeta})=\left.\partial_{\rho}\right|_{\rho=0} Z_{+}^{r+s}
\end{aligned}
$$

(recall the special choice made in Proposition 4.7). In the next computations we write

$$
\begin{aligned}
& Z_{-}^{n+s}=\left(z_{1-}, z_{2-}, z_{3-}, z_{2-}^{\prime}, z_{3-}^{\prime}\right)^{t} ; Z_{+}^{r+s}=\left(z_{1+}, z_{2+}, z_{3+}, z_{2+}^{\prime}, z_{3+}^{\prime}\right)^{t} \\
& \mathbb{Z}_{ \pm}=\left(\mathrm{z}_{1 \pm}, \mathrm{z}_{2 \pm}, \mathrm{z}_{3 \pm}, \mathrm{z}_{2 \pm}^{\prime}, \mathrm{z}_{3 \pm}^{\prime}\right)^{t} ; \mathbb{Z}=\left(\mathrm{z}_{1}, \mathrm{z}_{2}, \mathrm{z}_{3}, \mathrm{z}_{2}^{\prime}, \mathrm{z}_{3}^{\prime}\right)^{t}
\end{aligned}
$$

To determine the jump in variation of the profile, first take $Z=Z_{-}^{n+s}$ in (6.9)(a),(b), differentiate $\left.\partial_{\rho}\right|_{\rho=0}$; then do the same with $Z=Z_{+}^{r+s}$. Combining (a) and (b) equations in the 
obvious way gives

$$
\begin{aligned}
& \left(\mathcal{B}_{*}^{d d} \mathbf{z}_{b \pm}^{\prime}\right)^{\prime}=\left(\left(\mathcal{A}_{*}^{d}-\mathcal{B}^{d d}\left(C^{-1}\right)^{\prime}\right) \mathbf{z}_{b \pm}\right)^{\prime}-\sum^{d-1} i \hat{\eta}_{k}\left(\mathcal{B}_{*}^{d k} z_{b \pm}\right)^{\prime}+\hat{\lambda} \mathcal{A}_{*}^{0} z_{b_{ \pm}} \\
& -\sum_{j=1}^{d-1} i \hat{\eta}_{j} \mathcal{B}_{*}^{j d} z_{b \pm}^{\prime}+\sum_{j=1}^{d-1} i \hat{\eta}_{j}\left(\mathcal{A}_{*}^{d}-\mathcal{B}^{d d}\left(C^{-1}\right)^{\prime}\right) z_{b \pm} \\
& -Q\left\{\left(D^{d d}\left(\mathcal{K}{z_{3 \pm}}_{b^{\prime}}\right)^{\prime}-\left(P^{d} \mathcal{K}{z_{3 \pm}}_{3}+E_{*}^{d} \mathbf{z}_{b \pm}\right)^{\prime}+\sum_{k=1}^{d-1} i \hat{\eta}_{k}\left(D^{d k} \mathcal{K} z_{3 \pm}\right)^{\prime}\right\}\right. \\
& -Q\left\{-\sum_{j=1}^{d-1} i \hat{\eta}_{j}\left(P^{j} \mathcal{K}_{z_{3 \pm}}+E_{*}^{j} z_{b \pm}\right)+\sum_{j=1}^{d-1} i \hat{\eta}_{j} D^{j d}\left(\mathcal{K} z_{3 \pm}\right)^{\prime}\right\} \\
& +Q\left\{\hat{\lambda}\left(P^{0} \mathcal{K} z_{3 \pm}+E_{*}^{0} z_{b \pm}\right)\right\} .
\end{aligned}
$$

Observe that each line in (6.18) is a perfect derivative. Integrating the \pm equations $\int_{ \pm \infty}^{x}$ and recalling (4.74), we obtain

$$
\begin{aligned}
& \mathcal{B}_{*}^{d d} \mathbf{z}_{b \pm}^{\prime}(x, \hat{\zeta})=\left(\mathcal{A}_{*}^{d}-\mathcal{B}^{d d}\left(C^{-1}\right)^{\prime}\right) \mathbf{z}_{b \pm}-\sum^{d-1} i \hat{\eta}_{k} \mathcal{B}^{d k} \bar{V}^{\prime}+\hat{\lambda}\left(f^{0}(\bar{V})-f^{0}\left(V_{ \pm}\right)\right) \\
& +\sum_{j=1}^{d-1} i \hat{\eta}_{j}\left(f^{j}(\bar{V})-f^{j}\left(V_{ \pm}\right)-\mathcal{B}^{j d} \bar{V}^{\prime}\right) \\
& -Q\left\{D^{d d}\left(\mathcal{K} \mathbf{z}_{3 \pm}\right)^{\prime}-\left(P^{d} \mathcal{K} \mathbf{z}_{3 \pm}+E_{*}^{d} \mathbf{z}_{b \pm}\right)+\sum_{k=1}^{d-1} i \hat{\eta}_{k}\left(D^{d k} \bar{Y}^{\prime}\right\}\right. \\
& +Q\left\{\sum_{j=1}^{d-1} i \hat{\eta}_{j}\left(g^{j}(\bar{V}) \bar{Y}-g^{j}\left(V_{ \pm}\right) Y_{ \pm}-D^{j d} \bar{Y}^{\prime}\right)\right\} \\
& +Q\left\{\hat{\lambda}\left(g^{0}(\bar{V}) \bar{Y}-g^{0}\left(V_{ \pm}\right) Y_{ \pm}\right)\right\} .
\end{aligned}
$$

Next subtract the plus equation from the minus equation in (6.19) to get

$$
\begin{aligned}
& \mathcal{B}_{*}^{d d} \mathbf{z}_{b}^{\prime}=\left(\mathcal{A}_{*}^{d}-\mathcal{B}^{d d}\left(C^{-1}\right)^{\prime}\right) \mathbf{z}_{b}-Q\left\{D^{d d}\left(\mathcal{K} \mathbf{z}_{3}\right)^{\prime}-\left(P^{d} \mathcal{K} \mathbf{z}_{3}+E_{*}^{d} \mathbf{z}_{b}\right)\right\} \\
& +\sum_{j=1}^{d-1} i \hat{\eta}_{j}\left[f^{j}(\bar{V})\right]+\hat{\lambda}\left[f^{0}(\bar{V})\right]+\hat{\lambda} Q\left[g^{0}(\bar{V}) \bar{Y}\right]
\end{aligned}
$$

Finally, take components in (6.20) to obtain

$$
\begin{gathered}
0=\mathbf{z}_{1}+\left(\hat{\lambda}\left[f^{0}(\bar{V})\right]+i\left[f^{\hat{\eta}}(\bar{V})\right]+\hat{\lambda} Q\left[g^{0}(\bar{V}) \bar{Y}\right]\right)_{1} \\
\mathbf{z}_{2}^{\prime}=M_{1} \mathbf{z}_{1}+M_{2} \mathbf{z}_{2}-\left\{Q\left\{D^{d d}\left(\mathcal{K} \mathbf{z}_{3}\right)^{\prime}-\left(P^{d} \mathcal{K} \mathbf{z}_{3}+E_{*}^{d} \mathbf{z}_{b}\right)\right\}\right\}_{2} \\
+\left(\hat{\lambda}\left[f^{0}(\bar{V})\right]+i\left[f^{\hat{\eta}}(\bar{V})\right]+\hat{\lambda} Q\left[g^{0}(\bar{V}) \bar{Y}\right]\right)_{2} .
\end{gathered}
$$

We can now finish the proof of the Equivalence Theorem. 
Theorem 6.1. Under hypotheses (HO), (H1), (H2), (H3), (H4), (H5), (H6), and (H7), the RNS Evans function satisfies

$$
\begin{aligned}
D_{R N S}(\hat{\zeta}, \rho)= & \rho \beta_{2} \operatorname{det}\left(r_{-}^{1}(\hat{\zeta}), \ldots, r_{-}^{n-1}(\hat{\zeta}), \hat{\lambda}\left[f^{0}(\bar{V})\right]+i\left[f^{\hat{\eta}}(\bar{V})\right]+\hat{\lambda} Q\left[g^{0}(\bar{V}) \bar{Y}\right]\right)+o(\rho)= \\
& \rho \beta_{2} \Delta_{C J}(\hat{\zeta}, 1)+o(\rho),
\end{aligned}
$$

where

$$
\beta_{2}=(-1)^{r+s-1} \kappa^{-1} \gamma, \quad \kappa=\left.\left(\operatorname{det} A^{d} \operatorname{det} b^{d d} \operatorname{det} D^{d d}\right)\right|_{x=0},
$$

and $\gamma$ is the transversality coefficient given below by (6.26). The error is o( $\rho)$ uniformly for $\hat{\zeta} \in S_{+}^{d}=\{\hat{\zeta}:|\hat{\zeta}|=1, \hat{\gamma} \geq 0\}$.

Proof. From (6.7) we have at $x=0$

$$
\begin{aligned}
& \tilde{D}_{R N S}(\hat{\zeta}, \rho)=\operatorname{det}\left(Z_{-}^{1}, \ldots, Z_{-}^{n+s}, Z_{+}^{1}, \ldots, Z_{+}^{r+s}\right)= \\
& \operatorname{det}\left(Z_{-}^{1}, \ldots, Z_{-}^{n+s}, Z_{+}^{1}, \ldots,-\rho \mathbb{Z}+O\left(\rho^{2}\right)\right)=-\rho \operatorname{det}\left(Z_{-}^{1}, \ldots, Z_{-}^{n+s}, Z_{+}^{1}, \ldots, \mathbb{Z}+O(\rho)\right),
\end{aligned}
$$

where the second equality follows by subtracting the $(n+s)$-th column from the last column. Writing out the final term in (6.24) and using the row operations (6.14), (6.15), and (6.21) yields

$$
\begin{aligned}
-\rho \operatorname{det}\left(\begin{array}{cccccc}
z_{1-}^{1} & \cdots & z_{1-}^{n+s} & z_{1+}^{1} & \cdots & \mathrm{z}_{1}+O(\rho) \\
z_{2-}^{1} & \cdots & z_{2-}^{n+s} & z_{2+}^{1} & \cdots & \mathrm{z}_{2}+O(\rho) \\
z_{3-}^{1} & \cdots & z_{3-}^{n+s} & z_{3+}^{1} & \cdots & \mathrm{z}_{3}+O(\rho) \\
z_{2-}^{1^{\prime}} & \cdots & z_{2-}^{n+s^{\prime}} & z_{2+}^{1^{\prime}} & \cdots & \mathrm{z}_{2}^{\prime}+O(\rho) \\
z_{3-}^{1^{\prime}} & \cdots & z_{3-}^{n+s^{\prime}} & z_{3+}^{1^{\prime}} & \cdots & \mathrm{z}_{3}^{\prime}+O(\rho)
\end{array}\right)= \\
-\rho \operatorname{det}\left(\begin{array}{ccccc}
\left(r_{-}\right)_{1}+o(1) & O(\rho) & O(\rho) & -\left(\hat{\lambda}\left[f^{0}(\bar{V})\right]+i\left[f^{\hat{\eta}}(\bar{V})\right]+\hat{\lambda} Q\left[g^{0}(\bar{V}) \bar{Y}\right]\right)_{1}+O(\rho) \\
z_{2-} & z_{2-} & z_{2+} & \mathrm{z}_{2}+O(\rho) \\
z_{3-} & z_{3-} & z_{3+} & \mathrm{z}_{3}+O(\rho) \\
-\left(r_{-}\right)_{2}+o(1) & O(\rho) & O(\rho) & \left(\hat{\lambda}\left[f^{0}(\bar{V})\right]+i\left[f^{\hat{\eta}}(\bar{V})\right]+\hat{\lambda} Q\left[g^{0}(\bar{V}) \bar{Y}\right]\right)_{2}+O(\rho) \\
z_{3-}^{\prime} & z_{3-}^{\prime} & z_{3+}^{\prime} & \mathrm{z}_{3}^{\prime}+O(\rho)
\end{array}\right),
\end{aligned}
$$

where in the second determinant the columns have width $n-1, s+1, r+s-1$, and 1 respectively. Switching rows and columns we obtain (6.22), where $\gamma$ is the $(r+2 s) \times(r+2 s)$ subdeterminant

$$
\gamma=\operatorname{det}\left(\begin{array}{ll}
z_{2-} & z_{2+} \\
z_{3-} & z_{3+} \\
z_{3-}^{\prime} & z_{3+}^{\prime}
\end{array}\right)
$$

with columns drawn from the second and third columns of the second determinant in (6.25).

Remark 6.1. 1. The $o(1)$ and $O(\rho)$ terms in the above determinants are accounted for by Remark 4.6. The above argument shows that $D_{R N S}(\hat{\zeta}, \rho) / \rho$ is $C^{\infty}$ in $\hat{\gamma}>0, \rho>0$ with a continuous extension to $\hat{\gamma} \geq 0, \rho \geq 0$. 
2. The nonvanishing of $\beta_{2}$ for the physical RNS equations is discussed in section 8 of part 5.

Combining Theorems 5.1 and 6.1 we obtain:

Theorem 6.2 (Equivalence Theorem). Under assumptions (HO)-(H8) the ZND and RNS Evans functions satisfy

$$
\begin{aligned}
& \text { (a) } D_{Z N D}(\hat{\zeta}, \rho)=\rho \beta_{1} \Delta_{C J}(\hat{\zeta}, 1)+O\left(\rho^{2}\right) \\
& \text { (b) } D_{R N S}(\hat{\zeta}, \rho)=\rho \beta_{2} \Delta_{C J}(\hat{\zeta}, 1)+o(\rho),
\end{aligned}
$$

where $\beta_{1}$ (5.15) and $\beta_{2}(6.23)$ are transversality constants, $\hat{\zeta} \in S_{+}^{d}=\{(\lambda, \eta):|\lambda, \eta|=1, \Re \lambda \geq$ $0\}$, and $\rho=|\lambda, \eta|$. The errors are $O\left(\rho^{2}\right)$ (respectively, o $(\rho)$ ) uniformly for $\hat{\zeta} \in S_{+}^{d}$.

\section{Part 5. The physical equations}

\section{Evaluation of $\Delta_{C J}$}

In the previous sections we used abstract generalizations of the RNS, ZND, and CJ combustion models to streamline and clarify the proof of the Equivalence Theorem and its surrounding discussion. In this part, we restrict our attention to the important special cases of these generalizations where the functions $f^{j}, g^{j}$ of $(0.23)$ are those obtained from (0.15). We begin by finding zeros of $\Delta_{C J}$ as defined in (4.6). In the context of nonreacting gas dynamics, this calculation was originally carried out by Erpenbeck [E2]. (See also the more recent treatment in the expository appendix [JL] to [Z2].) Later in the context of the CJ model, this calculation was made by Majda and Rosales [MR] with a particular emphasis on the zeros $(\lambda=i \tau+\gamma, \eta)$ of $\Delta_{C J}$ with $\gamma=0$ and their role in the spontaneous formation of Mach stems in reacting (CJ) shock fronts. We revisit this calculation in the light of Theorem 6.2 which implies that these zeros have additional significance in that they contain information about both $D_{R N S}$ and $D_{Z N D}$. To make use of this information to make a conclusion like (0.13), we also verify that the transversality coefficients $\beta_{1}$ (defined in (5.15)) and $\beta_{2}$ (defined in $(6.23),(6.26)$ ) are nonvanishing. Finally, we look at the case of an ideal polytropic gas.

For simplicity and concreteness, we fix $d=2$ and $s=1$. That is, we consider the case of two space dimensions and a single one-step reaction. We suppose then that the detonation under consideration has Lax 4-shock structure. From (0.23) and (0.28) we immediately obtain (recall $V=(\rho, \mathbf{u}, T)^{\operatorname{tr}}$ and $\mathbf{u}=\left(u_{1}, u_{2}\right)^{\operatorname{tr}}$ ) for $V$ burnt that

$$
\begin{aligned}
& f^{0}(V)=\left(\rho, \rho u_{1}, \rho u_{2}, \rho\left(c_{v} T+|\mathbf{u}|^{2} / 2\right)\right)^{\operatorname{tr}} \\
& f^{1}(V)=\left(\rho u_{1}, \rho u_{1}^{2}+p, \rho u_{1} u_{2},\left(\rho\left(c_{v} T+|\mathbf{u}|^{2} / 2\right)+p\right) u_{1}\right)^{\operatorname{tr}},
\end{aligned}
$$

and

$$
f^{2}(V)=\left(\rho u_{2}, \rho u_{1} u_{2}, \rho u_{2}^{2}+p,\left(\rho\left(c_{v} T+|\mathbf{u}|^{2} / 2\right)+p\right) u_{2}\right)^{\operatorname{tr}} .
$$


Thus, the $4 \times 4$ matrices $a^{j}=d f^{j}$ of partial derivatives are easily calculated to be

$$
\begin{aligned}
a^{0}(V)= & \left(\begin{array}{cccc}
1 & 0 & 0 & 0 \\
u_{1} & \rho & 0 & 0 \\
u_{2} & 0 & \rho & 0 \\
c_{v} T+|\mathbf{u}|^{2} / 2 & \rho u_{1} & \rho u_{2} & c_{v} \rho
\end{array}\right), \\
a^{1}(V) & =\left(\begin{array}{cccc}
u_{1} & \rho & 0 \\
u_{1}^{2}+p_{\rho} & 2 \rho u_{1} & 0 & c_{v} p_{e} \\
u_{1} u_{2} & \rho u_{2} & \rho u_{1} & 0 \\
u_{1}\left(c_{v} T+|\mathbf{u}|^{2} / 2+p_{\rho}\right) & \rho u_{1}\left(c_{v} T+|\mathbf{u}|^{2} / 2\right)+p & \rho u_{1} u_{2} & c_{v} \rho u_{1}+u_{1} c_{v} p_{e}
\end{array}\right),
\end{aligned}
$$

and

$$
a^{2}(V)=\left(\begin{array}{cccc}
u_{2} & 0 & \rho & 0 \\
u_{1} u_{2} & \rho u_{2} & \rho u_{1} & 0 \\
u_{2}^{2}+p_{\rho} & 0 & 2 \rho u_{2} & c_{v} p_{e} \\
u_{2}\left(c_{v} T+|\mathbf{u}|^{2} / 2+p_{\rho}\right) & \rho u_{1} u_{2} & \rho u_{2}\left(c_{v} T+|\mathbf{u}|^{2} / 2\right)+p & c_{v} \rho u_{2}+u_{2} c_{v} p_{e}
\end{array}\right)
$$

We also note that

$$
Q=(0,0,0, q)^{\operatorname{tr}} \text { and } g^{0}\left(V_{+}\right)=\rho_{+} .
$$

Moreover, the jump condition (1.2) is equivalent to the following equations:

$$
\begin{aligned}
& {\left[\rho u_{2}\right]=0,} \\
& {\left[\rho u_{1} u_{2}\right]=0,} \\
& {\left[\rho u_{2}^{2}+p\right]=0,}
\end{aligned}
$$

and

$$
\left[\left(\rho\left(c_{v} T+|\mathbf{u}|^{2} / 2\right)+p\right) u_{2}\right]=-\rho_{+} u_{2+} q .
$$

Remark 7.1. We note that the jump conditions (7.8)-(7.11) simplify further due to the fact that the transverse velocity is zero. See Remark 1.2.

Specializing the definition of $\Delta_{C J}(4.6)$ to the case of interest, we find

$$
\Delta_{C J}(\zeta)=\operatorname{det}(r_{-}^{1}(\zeta), r_{-}^{2}(\zeta), r_{-}^{3}(\zeta), \underbrace{\lambda\left[f^{0}\right]+i \eta_{1}\left[f^{1}\right]+\lambda g^{0}\left(V_{+}\right) Q}_{\alpha})
$$

where $r_{-}^{j}(\zeta)=a^{d}\left(V_{-}\right) s_{-}^{j}(\zeta)$ and the collection $\left\{s_{-}^{1}, s_{-}^{2}, s_{-}^{3}\right\}$ spans the unstable eigenspace $F_{-}(\zeta)$ of

$$
H_{-}(\zeta)=-\left(a^{2}\right)^{-1}\left(\lambda a^{0}+i \eta_{1} a^{1}\right)\left(V_{-}\right)
$$

Equation (7.13), the definition of the $r_{-}^{j}$, and the fact that the $s_{-}^{j}$ span the unstable subspace of $H_{-}$imply that the $r_{-}^{j}$ are a basis for the stable subspace of

$$
\bar{H}_{-}(\zeta)=\left(\lambda a^{0}+i \eta_{1} a^{1}\right)\left(a^{2}\right)^{-1}\left(V_{-}\right) .
$$


Now, we let $\ell$ be a left eigenvector associated to the solitary unstable eigenvalue of $\bar{H}_{-}$. It follows that the expression for the CJ determinant can be rewritten as the dot product

$$
\Delta_{C J}(\zeta)=\ell \cdot \alpha
$$

The expressions in (7.1)-(7.3) and (7.7) allow us to write

$$
\alpha=\left(\begin{array}{c}
\lambda[\rho] \\
i \eta_{1}[p] \\
0 \\
\lambda\left[\rho\left(c_{v} T+|\mathbf{u}|^{2} / 2\right)\right]+\lambda \rho_{+} q
\end{array}\right)
$$

To find an expression for $\ell$, we take advantage of the fact (0.31) that the fluxes in the burnt gas are identical to the fluxes of the nonreacting Euler equations. Since we evaluate $\bar{H}_{-}$at the burnt state, it follows that we can use the calculations of [JL].

Lemma 7.1. The vector $\ell$ is a left eigenvector of $\bar{H}_{-}$with eigenvalue $\beta$ if and only if $\ell$ is a left eigenvector of $\left(\eta_{1} a^{1}+i \beta a^{2}\right)\left(a^{0}\right)^{-1}$ with eigenvalue $i \lambda$.

Proof. The proof is a simple calculation.

The importance of Lemma 7.1 is that the eigenvalues and eigenvectors of the matrix $\left(\eta_{1} a^{1}+\eta_{2} a^{2}\right)\left(a^{0}\right)^{-1}$ are computed in [JL]. The eigenvalues are

$$
\begin{aligned}
& \mathbf{u} \cdot \boldsymbol{\eta}-c|\boldsymbol{\eta}| \\
& \mathbf{u} \cdot \boldsymbol{\eta} \text { (double), } \\
& \mathbf{u} \cdot \boldsymbol{\eta}+c|\boldsymbol{\eta}|
\end{aligned}
$$

where $c$ is the sound speed (1.3), and we have used the notation $\boldsymbol{\eta}=\left(\eta_{1}, \eta_{2}\right)^{\text {tr }}$. We note that $\eta_{1}$ is the frequency variable dual to spatial variable $y_{1}$ while $\eta_{2}$ is an alternate label for $i \beta$. To simplify certain expressions, we shall use both labels simultaneously. To determine an expression for $\ell$, we need to identify which of (7.17)-(7.19) when set equal to $i \lambda$ yields $\Re \beta>0$. It's easy to discard the double eigenvalue (7.18), for, setting $i \lambda=\mathbf{u} \cdot \boldsymbol{\eta}$, we find

$$
i \lambda=i u_{2} \beta,
$$

since we take the transverse velocity $u_{1}$ to be zero by (1.14). Moreover, since $u_{2}<0$ by (1.15), equation (7.20) shows that $\Re \lambda$ and $\Re \beta$ have opposite signs. Since our interest is in $\Re \lambda \geq 0$, we do not find the unstable eigenvalue $\beta$ by this choice. It follows that

$$
\begin{aligned}
i \lambda & =\mathbf{u} \cdot \boldsymbol{\eta} \mp c|\boldsymbol{\eta}| \\
& =i u_{2} \beta \mp c \sqrt{\eta_{1}^{2}-\beta^{2}} .
\end{aligned}
$$

Remark 7.2. Some care needs to be taken with the square root in (7.22) to guarantee that the right hand side gives $\lambda$ as a continuous function of $\beta$ as $\beta$ varies in the right half plane. In this case, the branch cut should be taken along the positive real axis.

To determine which choice in (7.21) should be made, we consider the special case $\eta_{1}=0$ to find

$$
\Re \lambda=\left(u_{2} \mp c\right) \Re \beta .
$$


Since the burnt state is subsonic (Remark 1.1), we find that choosing + gives the unique choice of eigenvalues (7.17)-(7.19) for which $\Re \beta>0$. Therefore, from [JL], the desired left eigenvector $\ell$ is

$$
\begin{aligned}
\ell^{\operatorname{tr}} & =\left(\theta-\frac{c(\mathbf{u} \cdot \boldsymbol{\eta})}{|\boldsymbol{\eta}|}+\frac{\Gamma|\mathbf{u}|^{2}}{2}, c \frac{\boldsymbol{\eta}^{\operatorname{tr}}}{|\boldsymbol{\eta}|}-\Gamma \mathbf{u}^{\operatorname{tr}}, \Gamma\right) \\
& =\left(\theta-\frac{i c \beta u_{2}}{|\boldsymbol{\eta}|}+\frac{\Gamma \mid u_{2}^{2}}{2}, c \frac{\eta_{1}}{|\boldsymbol{\eta}|}, \frac{i c \beta u_{2}}{|\boldsymbol{\eta}|}-\Gamma u_{2}, \Gamma\right),
\end{aligned}
$$

where $\theta=p_{\rho}-\Gamma c_{v} T$, and $\Gamma=p_{e} / \rho$ is the Gruneisen coefficient. Thus we now have the basic ingredients $\alpha$ and $\ell$ to compute $\Delta_{C J}$. Before doing so, we note a pair of useful relations that follow directly from our definitions above:

$$
\begin{array}{r}
\lambda=\beta u_{2}-i c \sqrt{\eta_{1}^{2}-\beta^{2}}, \\
\beta=\frac{\lambda u_{2}-c \sqrt{\lambda^{2}-\eta_{1}^{2}\left(u_{2}^{2}-c^{2}\right)}}{u_{2}^{2}-c^{2}} .
\end{array}
$$

We note that in (7.27) the standard branch of the square root is used. Finally, we are ready to compute $\Delta_{C J}$. We combine (7.16) and (7.25) using (7.15) to get

$$
\Delta_{C J}=\lambda[\rho]\left\{\theta-\frac{i c \beta u}{|\boldsymbol{\eta}|}+\Gamma \frac{u_{2}^{2}}{2}\right\}+i \eta_{1}[p]\left\{\frac{c \eta_{1}}{|\boldsymbol{\eta}|}\right\}+\lambda \Gamma[\mathcal{E}]+\lambda \Gamma \rho_{+} q
$$

where we have used the shorthand $\mathcal{E}=\rho\left(c_{v} T+u_{2}^{2} / 2\right)$. We note that everything in (7.28) is evaluated at the burnt state $V_{-}$unless it is otherwise indicated by the jump notation [.] or by a subscript + . Equation (7.28) gives $\Delta_{C J}$ as a function of $\lambda$ and $\eta_{1}$. Henceforth, we omit the subscript 2 on $u_{2}$ and the subscript 1 on $\eta_{1}$. We define

$$
\tilde{\mathcal{R}}=\theta+\Gamma \frac{u^{2}}{2}+\Gamma \frac{[\mathcal{E}]+\rho_{+} q}{[\rho]}
$$

and we use the fact from the jump conditions (7.8) and (7.10) that the jump in the pressure can be written as $[p]=u_{+} u[\rho]$ to rewrite the expression for $\Delta_{C J}$ as

$$
\Delta_{\mathrm{CJ}}=[\rho]\left\{\lambda \tilde{\mathcal{R}}-\lambda \frac{i c \beta u}{|\boldsymbol{\eta}|}+\frac{i c u_{+} u \eta^{2}}{|\boldsymbol{\eta}|}\right\}
$$

Now we define

$$
\omega=\frac{\lambda}{|u|}, \quad M=\frac{|u|}{c}, \quad r=\frac{\rho}{\rho_{+}}=1 / \rho_{+} \quad\left(\text { We set } \rho_{-}=1\right. \text { as in Remark 2.1). }
$$


Thus, we find by some elementary manipulations that

$$
\begin{aligned}
\Delta_{C J} & =|u|[\rho] c^{2}\left\{\frac{\omega \tilde{\mathcal{R}}}{c^{2}}+\frac{i M \beta \omega}{|\boldsymbol{\eta}|}+\frac{i \eta^{2} M r}{|\boldsymbol{\eta}|}\right\} \\
& =|u|[\rho] c^{2}\left\{\frac{\omega \tilde{\mathcal{R}}}{c^{2}}+\frac{\beta \omega}{\omega+\beta}+\frac{\eta^{2} r}{\omega+\beta}\right\} \\
& =\frac{|u|[\rho] c^{2}}{\omega+\beta}\left\{\frac{\tilde{\mathcal{R}}}{c^{2}} \omega(\omega+\beta)+\beta \omega+\eta^{2} r\right\} .
\end{aligned}
$$

Now, we define

$$
\begin{aligned}
& s(\omega)=\omega+\sqrt{M^{2} \omega^{2}+\left(1-M^{2}\right) \eta^{2}}, \\
& \tilde{\kappa}=\frac{\tilde{\mathcal{R}}}{c^{2}}+1,
\end{aligned}
$$

and we note that

$$
\omega+\beta=\frac{s(\omega)}{1-M^{2}} .
$$

We can now rewrite our expression for $\Delta_{C J}$ using $\tilde{\kappa}$ and $s(\omega)$. We find

$$
\begin{aligned}
\Delta_{C J} & =\frac{|u|[\rho] c^{2}}{\omega+\beta}\left\{\frac{\tilde{\kappa} \omega s(\omega)}{1-M^{2}}+\eta^{2} r-\omega^{2}\right\} \\
& =\frac{|u|[\rho] c^{2} r}{s(\omega)}\left\{r^{-1} \tilde{\kappa} \omega s(\omega)+\left(1-M^{2}\right)\left(\eta^{2}-r^{-1} \omega^{2}\right)\right\}
\end{aligned}
$$

From (7.39) and nonvanishing of $|u|[\rho] c^{2} r$ it follows that zeros of $\Delta_{C J}$ are exactly those of the analytic expression

$$
G(\omega, \eta):=r^{-1} \tilde{\kappa} \omega s(\omega)+\left(1-M^{2}\right)\left(\eta^{2}-r^{-1} \omega^{2}\right) .
$$

Recall that our interest is in zeros $(\lambda, \eta)$ of $\Delta_{C J}$ which lie in the set

$$
S_{+}^{2}:=\{(\lambda, \eta) \in \mathbb{C} \times \mathbb{R}:|(\lambda, \eta)|=1, \Re \lambda \geq 0\} .
$$

In particular, $(\omega, \eta)=(0,0) \notin S_{+}^{2}$. We consider the cases $\eta=0$ and $\eta \neq 0$ separately. In the case $\eta=0,(7.40)$ simplifies to

$$
|u|[\rho] c^{2}[\tilde{\kappa}-1+M] \omega .
$$

There are therefore two possibilities in this case:

- if $\tilde{\kappa}=1-M$ then $\Delta_{C J}(\omega) \equiv 0$,

- if $\tilde{\kappa} \neq 1-M$ then $\Delta_{C J}(\omega)=0$ only for $\omega=0$.

In the case $\eta \neq 0$, we may set $\eta=1$ by homogeneity. The quantity $s(\omega)$ is then given as

$$
s(\omega):=\omega+\sqrt{M^{2} \omega^{2}+\left(1-M^{2}\right)},
$$

where the square root has positive real part $(s(\omega) \neq 0$ for all $\omega$ with real part $\geq 0)$. Thus from (7.40), The zeros of $\Delta_{C J}$ in the closed right half plane are then the same as those of 
the function

$$
\begin{aligned}
G(\omega) & =r^{-1} \tilde{\kappa} \omega s(\omega)+\left(1-M^{2}\right)\left(1-r^{-1} \omega^{2}\right) \\
& =\left(1-M^{2}\right)+r^{-1}\left[\tilde{\kappa}-1+M^{2}\right] \omega^{2}+r^{-1} \tilde{\kappa} M \omega \sqrt{\omega^{2}+\beta^{2}},
\end{aligned}
$$

where $\beta^{2}=\left(1-M^{2}\right) / M^{2}$ and the square root has positive real part. This is the same expression that was analyzed in the non-reactive case. A detailed winding number analysis of this function as in [JL] gives the following cases:

- Case Ia: $1-M-\tilde{\kappa}<0 \& M^{2}-r^{-1}\left(\tilde{\kappa}-1+M^{2}\right) \geq 0$ : $G$ has no roots in the open right half plane and exactly two roots on the imaginary axis.

- Case Ib: $1-M-\tilde{\kappa}<0 \& M^{2}-r^{-1}\left(\tilde{\kappa}-1+M^{2}\right)<0$ : $G$ has no roots in the closed right half plane.

- Case II: $1-M-\tilde{\kappa}>0: G$ has no roots on the imaginary axis and exactly one root in the open right half-plane.

- Case III: $1-M-\tilde{\kappa}=0: G$ has no roots in the closed right half-plane.

These conclusions hold under the assumption that $M<1$. (Recall that the the burnt state $V_{-}$is subsonic.) As $r^{-1}>0$ we have that $\tilde{\kappa}>1-M^{2}+M^{2} r$ implies $\tilde{\kappa}>1-M$, whence Case Ib occurs if and only if $\tilde{\kappa}>1-M^{2}+M^{2} r$.

Putting the various cases together we thus have the following breakdown for roots $(\lambda, \eta)$ of $\Delta_{C J}$ :

- $\tilde{\kappa} \leq 1-M: \exists$ a root $(\lambda, \eta) \in S_{+}^{2}$ with $\Re \lambda>0$,

- $1-M<\tilde{\kappa} \leq 1-M^{2}+M^{2} r: \exists$ at least one root $(\lambda, \eta) \in S_{+}^{2}$ with $\Re \lambda=0$, but no root with $\Re \lambda>0$,

- $\tilde{\kappa}>1-M^{2}+M^{2} r: \exists \operatorname{no} \operatorname{root}\left(\lambda, \xi_{2}\right) \in S_{+}^{2}$.

To express these criteria in terms of physical quantities we note that

$$
\tilde{\kappa}=2+\Gamma M^{2}(1-r) .
$$

(To obtain (7.43), we use the relations

$$
\theta=c^{2}-\Gamma\left(e+\frac{p}{\rho}\right), \quad \frac{[\mathcal{E}]+\rho_{+} q}{[\rho]}=\left(e+\frac{p}{\rho}\right)+\left(\frac{1}{2}-r\right) u^{2},
$$

which follow from repeated use of the Rankine-Hugoniot relations.) Applying this in the characterization above we conclude that a strong detonation front of the last family is

- strongly low frequency unstable if and only if

$$
\Gamma \geq\left(\frac{1}{r-1}\right)\left(\frac{1+M}{M^{2}}\right)
$$

- weakly low frequency stable if and only if

$$
\left(\frac{1}{r-1}\right) \frac{1}{M^{2}}-1 \leq \Gamma<\left(\frac{1}{r-1}\right)\left(\frac{1+M}{M^{2}}\right),
$$

- uniformly low frequency stable if and only if

$$
\Gamma<\left(\frac{1}{r-1}\right) \frac{1}{M^{2}}-1 \text {. }
$$

Remark 7.3. The above argument can be repeated in the case $d=3$ with only cosmetic changes due to the fact that there are now two transverse spatial directions. The same stability criteria are obtained at the end. 


\section{The transversality COEFFicients $\beta_{1}$ And $\beta_{2}$}

8.1. ZND. In the case $s=1, d=2$ that we are considering, the transversality coefficient $\beta_{1}$ of (5.15) simplifies to

$$
\beta_{1}=-\left.g^{2}\left(V_{*}\right) \kappa \mathrm{y}^{n}\right|_{x=0^{-}, \zeta=0},
$$

where now

$$
\begin{aligned}
g^{2}\left(V_{*}\right) & =\left.\left(\rho u_{2}\right)\right|_{x=0^{-}}, \\
\kappa & =\left(\operatorname{det} a^{2}\right)\left(\operatorname{det}\left(a^{2}+N\right)\right)^{-1}, \\
\mathrm{y}^{n} & =\text { last component of }\left.\tilde{w}^{\prime}\right|_{x=0^{-}},
\end{aligned}
$$

and $\tilde{w}$ is the stationary ZND profile described in section 1.3. The expression for $\mathrm{y}^{n}$ in (8.4) is obtained since $\mathrm{y}^{n}$ is one component of the distinguished fast mode (4.33). Now, we note that

$$
\left.\operatorname{det} a^{2}\right|_{x=0^{-}}=u_{2 *}^{2}\left(u_{2 *}^{2}-c_{*}^{2}\right),
$$

which is nonzero provided that the von Neumann shock is not sonic.

The matrix $N(5.1)$ is defined by

$$
N v=Q d_{V} g^{2}\left(V_{*}\right) v
$$

since the jump conditions imply $Y_{*}=1$. Using $g^{2}(V)=\rho u_{2}$, it is easy to see from the form of $Q$ in (7.7) that

$$
N=q\left(\begin{array}{cccc}
0 & 0 & 0 & 0 \\
0 & 0 & 0 & 0 \\
0 & 0 & 0 & 0 \\
u_{2} & 0 & \rho & 0
\end{array}\right)
$$

Moreover, from (7.6) and multilinearity of the determinant, we can compute

$$
\operatorname{det}\left(a^{2}+N\right)=\operatorname{det} a^{2}-q u_{2}\left|\begin{array}{ccc}
0 & \rho & 0 \\
\rho u_{2} & \rho u_{1} & 0 \\
0 & 2 \rho u_{2} & c_{v} p_{e}
\end{array}\right|-q \rho\left|\begin{array}{ccc}
u_{2} & 0 & 0 \\
u_{1} u_{2} & \rho u_{2} & 0 \\
u_{2}^{2}+p_{\rho} & 0 & c_{v} p_{e}
\end{array}\right|=\operatorname{det} a^{2}
$$

It follows that $\kappa=1$. Finally, we need to look at $\mathrm{y}^{n}$ which is the final component of the ZND profile $\tilde{w}=(\tilde{V}, \tilde{Y})$ which is a solution of the ODE (1.8)

$$
\left(\begin{array}{c}
f^{2}(\tilde{V}) \\
\left.g^{2}(\tilde{V}) \tilde{Y}\right)
\end{array}\right)^{\prime}=\left(\begin{array}{c}
Q K \psi(\tilde{V}) \tilde{Y}) \\
-K \psi(\tilde{V}) \tilde{Y}
\end{array}\right)
$$

Therefore,

$$
a^{2}(\tilde{V}) \tilde{V}^{\prime}+Q d_{V} g^{2}(\tilde{V}) \tilde{V}^{\prime} \tilde{Y}+Q g^{2}(\tilde{V}) \tilde{Y}^{\prime} \equiv 0
$$

which we rewrite as

$$
\left(a^{2}+N\right) \tilde{V}^{\prime}+Q g^{2}(\tilde{V}) \tilde{Y}^{\prime} \equiv 0 .
$$

Then invertibility of $a^{2}+N$ implies that if $\left.\tilde{Y}^{\prime}\right|_{x=0^{-}}=0$, then $\left.\tilde{V}^{\prime}\right|_{x=0^{-}}=0$ also. In this case it follows from (8.9) that

$$
\left.\psi(\tilde{V})\right|_{x=0^{-}}=0
$$


which implies that the temperature at the left of the shock is below ignition temperature, a contradiction.

Remark 8.1. The above argument for the nonvanishing of $\operatorname{det} a^{d}$ works equally well in the case $d=3$. One finds simply that $\operatorname{det} a^{3}=u_{3 *}^{3}\left(u_{3 *}^{2}-c_{*}^{2}\right)$ which is nonzero under a nonsonicity assumption. Also, we find as before that $\operatorname{det}\left(a^{3}+N\right)=\operatorname{det} a^{3}$, and thus the same reasoning as above shows that $\mathrm{y}^{n}$ is nonzero given that the temperature after the von Neumann shock is above ignition.

We note that a computation similar to (8.8) shows that $\kappa=1$ in the multistep $(s>1)$ case as well.

8.2. RNS. Next we examine the coefficient $\beta_{2}$ that appears in the low frequency expansion of $D_{R N S}$ (6.27). As above we concentrate on the simplest interesting case: $s=1, d=2$. (Thus in our labeling $n=4$ and $r=3$.) From (6.23) we have that

$$
\beta_{2}=(-1)^{3+1-1} \kappa^{-1} \gamma
$$

It is straightforward to check that $\kappa \neq 0$, so the only way that $\beta_{2}$ may vanish is if $\gamma$ is zero. Using (6.26) in the current setting (physical equations $d=2, s=1$ ), we find that

$$
\gamma=\operatorname{det}\left(\begin{array}{lllll}
z_{2-}^{4} & z_{2-}^{5} & z_{2+}^{1} & z_{2+}^{2} & z_{2+}^{3} \\
z_{3-}^{4} & z_{3-}^{5} & z_{3+}^{1} & z_{3+}^{2} & z_{3+}^{3} \\
z_{3-}^{4^{\prime}} & z_{3-}^{5^{\prime}} & z_{3+}^{1^{\prime}} & z_{3+}^{2^{\prime}} & z_{3+}^{3^{\prime}}
\end{array}\right)
$$

where $z_{2 \pm}^{j} \in \mathbb{R}^{3} ; z_{3 \pm}^{j}, z_{3 \pm}^{j^{\prime}} \in \mathbb{R}$ so that $\gamma$ is defined as the determinant of a $5 \times 5$ matrix.

Let us briefly indicate why nonvanishing of $\gamma$ is equivalent to transversality of the intersection of the stable/unstable manifolds of the traveling wave ODE at the end states $w_{+} / w_{-}$ respectively. The columns of the matrix in (8.13) form bases for the tangent manifolds along the profile $\bar{w}$ of the stable/unstable manifolds at $w_{+} / w_{-}$. (Recall that the second column is the distinguished fast mode which vanishes at both infinities.) Thus, $\gamma \neq 0$ indicates that these tangent spaces span all of the phase space $\mathbb{R}^{5}$. That is, the intersection is tranverse.

We note that the phase space is five dimensional due to the real viscosity. The lack of a second-order term in the conservation equation implies that integration of that equation in the traveling wave ODE (1.6) yields simply that the mass flux $\rho u_{2} \equiv$ constant (1.7). Therefore, there is one dimension for each remaining conservation equation ( 2 momentum, 1 energy) and two dimensions for the single second-order reaction equation which cannot be integrated. However, we may take advantage of the fact (1.14) that we may take the transverse velocity to satisfy $u_{1} \equiv 0$; we thus reduce the number of unknowns by one. The resulting four-dimensional phase space is precisely that studied by Gasser-Szmolyan [GS]. Provided the dissipative coefficients are small enough so that the construction of [GS] is valid, it is shown in [LyZ] that the intersection of the stable/unstable manifolds is transverse.

Remark 8.2. The case $d=3$ presents no additional difficulty since both transverse velocities may be taken to be identically zero yielding the same reduction as above. However, in the interesting case $s>1$, the dimension of the phase space increases and the problem becomes more difficult. 


\section{The CASE OF AN IDEAL POLYTROPIC GAS}

Finally, we consider the case of an ideal polytropic gas. That is, we suppose that the pressure (0.18) satisfies

$$
p(\rho, e)=\Gamma \rho e
$$

where $\Gamma$, the Gruneisen coefficient as above, is now a fixed constant. In this case we see directly from the Rankine-Hugoniot conditions and the fact that the pressure is positive that

$$
(r-1)(\Gamma+1) M^{2}=1-\frac{p_{+}}{p}<1
$$

It follows that

$$
\Gamma<\left(\frac{1}{r-1}\right) \frac{1}{M^{2}}-1,
$$

which implies that the detonation is stable in the sense that $\Delta_{C J}$ has no zeros with $\gamma \geq 0$.

Remark 9.1. This calculation is identical to the calculation that an ideal gas shock is uniformly stable. See [Ma1, Ma2].

\section{Part 6. Appendix: Well-posedness of the combustion equations}

Here we describe how to put the physical ZND and RNS equations into Kawashima normal form. Derived in [KaSh2] for the nonreactive Navier-Stokes equations, this normal form is a highly symmetric and partially decoupled form of the equations that allows a simple proof of short time existence for the initial value problem with sufficiently regular initial data.

Let

$$
\sum_{j=0}^{3} F^{j}(w)_{y_{j}}=\sum_{j, k=1}^{3}\left(B^{j k}(w) w_{y_{k}}\right)_{y_{j}}+R(w)
$$

be the physical one-step RNS equations in dimension $d=3$, where $F^{j}, B^{j k}$, and $R$ may be read off from the system (0.15a), (0.15b), (0.20), (0.15d).

Define a $6 \times 6$ Kawashima symmetrizer for $(9.4)$ as follows:

$$
S(w)=\frac{1}{T}\left(\begin{array}{cccc}
\frac{p_{\rho}}{\rho} & 0 & 0 & 0 \\
-\mathbf{u}^{t} & I & 0 & 0 \\
\frac{-\left(e-\frac{|\mathbf{u}|^{2}}{2}\right)}{T} & \frac{-\mathbf{u}}{T} & \frac{1}{T} & 0 \\
-Y & 0 & 0 & 1
\end{array}\right) .
$$

$S(w)$ is obtained from the symmetrizer defined in [KaSh2] simply by adding a row and column.

Let $y_{s}=\left(y_{1}, y_{2}, y_{3}\right)$. Performing the $y_{j}$ derivatives on the left and right in (9.4) and then multiplying the resulting equation on the left by $S$ we obtain the normal form:

$$
\sum_{j=0}^{3} \tilde{A}^{0}(w) w_{y_{j}}=\sum_{j, k=1}^{3} \tilde{B}^{j k}(w) w_{y_{j} y_{k}}+\tilde{g}\left(w, \partial_{y_{s}} w\right)+S(w) R(w)
$$


where

$$
\tilde{A}^{j}=S A^{j}, \tilde{B}^{j k}=S B^{j k}, \tilde{g}\left(w, \partial_{y_{s}} w\right)=S\left(B^{j k}\right)_{y_{j}} w_{y_{k}} .
$$

The coefficients in (9.6) have the following properties:

1. $\tilde{A}^{0}=\left(\begin{array}{cc}\tilde{A}_{11}^{0} & 0 \\ 0 & \tilde{A}_{22}^{0}\end{array}\right)$ is symmetric and positive definite $\left(\tilde{A}_{11}^{0}\right.$ is $\left.1 \times 1\right)$;

2. The matrices $\tilde{A}^{j}, \tilde{B}^{j k}$ are symmetric;

3. $\tilde{B}^{j k}=\left(\begin{array}{cc}0 & 0 \\ 0 & \tilde{\mathbb{B}}^{j k}\end{array}\right)$ with $\sum_{j, k=1}^{3} \tilde{\mathbb{B}}^{j k} \xi_{j} \xi_{k} \geq C|\xi|^{2}$;

4. $\tilde{g}\left(w, \partial_{y_{s}} w\right)=\left(0, \tilde{g}_{2}\left(w, \partial_{s} w\right)\right)$.

It is now easy to check that short time existence and uniqueness of solutions to the initial value problem for sufficiently regular initial data follows by standard Friedrichs estimates and Picard iteration (see, e.g., [Ma3]).

The same symmetrizer $S(w)$ works for the ZND equations. The ZND normal form is given by (9.6) with the $\tilde{B}^{j k}$ and $\tilde{g}$ set equal to zero.

Remark 9.2. 1. We are not aware of any other results on local well-posedness for the RNS and ZND models in several space dimensions.

2. For the ZND model Chen and Wagner [CW] have recently proved a Glimm-type existence result in one dimension for small BV solutions. For the one dimensional RNS model without species diffusion, Chen, Hoff, and Trivisa [CHT] have established global existence, regularity, and large-time results. It is noteworthy that their analysis allows for the pressure and internal energy to depend on the mass fraction of reactant.

\section{Acknowledgment}

We have tried in Remark 0.3 to make clear the substantial influence of [ZS] and Appen$\operatorname{dix} \mathrm{A} 3$ of $[\mathrm{Z}]$ on this work. We would like to thank Kevin Zumbrun for numerous helpful conversations. Research of H.K.J. was supported by NSF grant \#0206631, G.L. was supported by a University of Michigan Rackham Faculty Fellowship, and M.W. by NSF grants DMS-0070684 and DMS-0401252.

\section{REFERENCES}

[AT] Abouseif, G. and Toong, T., Theory of unstable one dimensional detonations, Combustion and Flame, 45, 1982, 67-94.

[BM1] Burlioux, A. and Majda, A., Theoretical and numerical structure for unstable two dimensional detonations, Combustion and Flame, 90, 1992, 211-229.

[BM2] Burlioux, A. and Majda, A., Theoretical and numerical structure of unstable detonations, Phil. Trans. Roy. Soc. London A., 350, 1995, 29-68.

[BN] Buckmaster, J. and Nevis, J., One dimensional detonation stability-the spectrum for infinite activation energy, Physics of Fluids, 31, 1988, 3571-3575.

[Ch] Chandrasekhar, S., Hydrodynamic and hydromagnetic stability, Clarendon Press, Oxford, 1961.

[CW] Global entropy solutions to exothermically reacting, compressible Euler equations, J. Diff. Eqns., 191, 2003, 277-322.

[CHT] Global solutions to a model for exothermically reacting, compressible flows with large discontinuous initial data, Arch. Rat. Mech. Analysis, 166, 2003, 321-358. 
[CF] Courant, R. and Friedrichs, K., Supersonic flow and shock waves, Springer-Verlag, New York, 1976.

[Co] Coppel, W. A., Stability and asymptotic behavior of differential equations, D.C. Heath, Boston.

[CMR] Colella, P., Majda, A., and Roytburd, V., Theoretical and numerical structure for reacting shock waves, SIAM J. Sci. Stat. Comput., 7, 1986, 1059-1080.

[CP] Chazarain, J. and Piriou, A., Introduction to the theory of linear partial differential equations Studies in Mathematics and its Applications, 14, North-Holland, Amsterdam-New York, 1982. .

[DR] Drazin, P.G, and Reid, W.H., Hydrodynamic Stability, Cambridge Univ. Press, Cambridge, 1981.

[E1] Erpenbeck, J.J., Stability of steady-state equilibrium detonations, Physics of Fluids, 5, 1962, 11811187.

[E2] Erpenbeck, J.J., Stability of step shocks, Physics of Fluids, 5, 1962, 1181-1187.

[E3] Erpenbeck, J.J., Stability of idealized one reaction detonations, Physics of Fluids, 7, 1964, 684-696.

[E4] Erpenbeck, J.J., Steady detonations in idealized two reaction systems, Physics of Fluids, 7, 1964, 1424-1432.

[E5] Erpenbeck, J.J., Detonation stability for disturbances of small transverse wavelength, Physics of Fluids, 9, 1966, 1293-1306.

[E6] Erpenbeck, J.J., Nonlinear theory of unstable one dimensional detonations, Physics of Fluids, 10, 1967, 274-289.

[FD] Fickett, W. and Davis, W., Detonation: Theory and Experiment, Univ. California Press, Berkeley, 1979.

[FW] Fickett, W. and Wood, W., Flow calculations for pulsating one dimensional detonations, Physics of Fluids, 9, 1966, 903-916.

[GS] Gasser, I. and Szmolyan, P., A geometric singular perturbation analysis of detonation and deflagration waves, SIAM J. Math. Anal., 24, 1993, 968-986.

[G] Gardner, R., On the detonation of a combustible gas, Trans. Amer. Math. Soc., 277, 1983, 431-468.

[GZ] Gardner, R. and Zumbrun, K., The gap lemma and geometric criteria instability of viscous shock profiles, Comm. Pure Appl. Math. 51. 1998, 797-855.

[GMWZ1] Gues, O., Metivier, G., Williams, M., and Zumbrun, K., Multidimensional viscous shocks I: degenerate symmetrizers and long time stability, to appear in Journal of the AMS, available at http://www.math.unc.edu/Faculty/williams/

[GMWZ2] Gues, O., Metivier, G., Williams, M., and Zumbrun, K., Multidimensional viscous shocks II: the small viscosity limit, Comm. Pure Appl. Math., 57, 2004, 141-218.

[GMWZ3] Guès, O., Métivier, G., Williams, M., and Zumbrun, K., Existence and stability of multidimensional shock fronts in the vanishing viscosity limit, to appear in Arch. Rat. Mech. Analysis.

[GMWZ4] Guès, O., Métivier, G., Williams, M., and Zumbrun, K., Navier-Stokes regularization of multidimensional Euler shocks, in preparation.

[HR] Hesaaraki, M. and Razani, A., Detonative Travelling Waves for Combustions, Applicable Analysis, 77, 2001, 405-418.

[JL] Jenssen, H.K. and Lyng, G., Evaluation of the Lopatinski Determinant for multi-dimensional Euler equations, appendix to [Z2].

[Kat] Kato, T., Perturbation Theory for Linear Operators, Springer-Verlag, Berlin, 1995.

[Ka] Kawashima, S, Systems of hyperbolic-parabolic type with applications to the equations of magnetohydrodynamics, Ph.D. thesis, Kyoto Univ., 1983.

[KaSh1] Kawashima, S. and Shizuta, Y., Systems of equations of hyperbolic-parabolic type with applications to the discrete Boltzmann equation, Hokkaido Math. J., 14, 1985, 249-275.

[KaSh2] Kawashima, S. and Shizuta, Y., On the normal form of the symmetric hyperbolic-parabolic systems associated with the conservation laws, Tohoku Math. J., 1988, 449-464.

[KS] Kapitula, T. and Sandstede, B., Stability of bright solitary-wave solutions to perturbed nonlinear Schrödinger equations, Phys. D. 124. 1998, 58-103.

[K] H.O. Kreiss, Initial boundary value problems for hyperbolic systems, Comm. Pure Appl. Math. 23 (1970) 277-298.

[LS] Lee, H. and Stewart, D., Calculation of linear detonation instability: one dimensional instability of plane detonation, J. Fluid Mech., 216,1990, 103-132. 
[LyZ] Lyng, G. and Zumbrun, K., One-dimensional stability of viscous strong detonation waves, Arch. Rational Mech. Anal., 173, 2004, 213-277.

[Ma1] A. Majda, The stability of multi-dimensional shock fronts - a new problem for linear hyperbolic equations. Mem. Amer. Math. Soc. 275 (1983).

[Ma2] Majda, A., The existence of multidimensional shock fronts, Mem. Amer. Math. Soc. No. 281, AMS, Providence, 1983.

[Ma3] Majda, A., Compressible fluid flow and systems of conservation laws in several space variables, Springer-Verlag, New York, 1984.

[MR] Majda, A. and Rosales, R., A theory for spontaneous Mach stem formation in reacting shock fronts, I. The basic perturbation analysis, SIAM J. Appl. Math., 43, 1983, 1310-1334.

[MZ1] Métivier, G. and Zumbrun, K., Large viscous boundary layers for noncharacteristic nonlinear hyperbolic problems, to appear in Memoirs of the AMS, available at http://www.math.ubordeaux.fr/ metivier/Preprints.html

[MZ2] Métivier, G. and Zumbrun, K., Symmetrizers and continuity of stable subspaces for parabolichyperbolic boundary value problems, Disc. Cont. Dyn. Syst., 11, 2004, 205-220.

[Sh] Short, M., Multidimensional linear stability of a detonation wave at high activation energy, SIAM J. Appl. Math., 57,1997, 307-326.

[SS] Short, M. and Stewart, D.S., The multidimensional stability of weak heat-release detonations, J. Fluid Mech., 382, 1999, 109-135.

[SZ] Serre, D. and Zumbrun, K., Boundary layer stability in the real vanishing viscosity limit, Comm. Math. Physics, 221, 2001, 267-292.

[Wa] Wagner, D., The existence and behavior of viscous structure for plane detonation waves, SIAM J. Math. Anal., 20, 1989, 1035-1054

[W] Williams, M., Stability of multidimensional viscous shocks CIME lectures, to appear in Springer Lecture Notes, 2003.

[Wi] Williams, F., Combustion Theory, Benjamin-Cummings, Menlo Park, 1985.

[ZS] Zumbrun, K. and Serre, D. Viscous and inviscid stability of multidimensional planar shock fronts, Indiana Univ. Math. J., 48, 1999, 937-992.

[Z] Zumbrun, K., Multidimensional stability of planar viscous shock waves, Advances in the theory of shock waves, 304-516. Progress in Nonlinear PDE, 47, Birkhäuser, Boston, 2001.

[Z2] Zumbrun, K., Stability of large-amplitude shock waves of compressible Navier-Stokes equations, Handbook of Mathematical Fluid Dynamics III, S. Friedlander and D. Serre eds., North-Holland, Amsterdam, 2004. 